\title{
Les figures du décorateur français au XXe siècle, une histoire de mots et de pratiques'
}

\author{
The image of French decorators in the 20th century, a history of words and \\ practices
}

http://dx.doi.org/10.1590/1982-02672019v27e01

\section{BEATRICE GRONDIN²}

https: / / orcid.org/0000-000 1-9098-4266

Université Paris Nanterre / Paris, France

RESUMÉ: L'évolution sémantique du mot décorateur révèle les processus identitaires en jeu dans la construction des figures d'artistes. A l'orée du XXe siècle en France, les nouvelles valeurs de ce vocable trouvent leur origine dans l'affirmation du statut d'artiste créateur et dans la consécration de la synthèse des arts. Une figure idéale d'artiste décorateur se dessine sans pour autant renvoyer à une catégorie professionnelle stable. En effet, la réception critique ne fait pas consensus autour des compétences, entre savoir et savoir-faire, collaboration indépendante et maîtrise d'oeuvre, vision esthétique et dessin technique, conception sur mesure et en série. De l'ensemblier à l'architecte d'intérieur, le terme décorateur ne parvient à aucune définition sociale et professionnelle propre. La conception de la décoration de style français moderne et la nécessité de répondre aux besoins réels de la société divisent les créateurs. Les principes rationalistes de l'aménagement, défendus par l' Union des artistes modernes (UMA) en 1929, mettent définitivement en crise l'identité du décorateur ornementaliste. La dépréciation sémantique qui se poursuit pendant les Trente Glorieuses aboutit à des revendications professionnelles autour de "l'architecte spécialisé". Des groupements et syndicats comme l'Union des Créateurs d'ensembles et de modèles (UADCE) et le Syndicat des créateurs d'architectures intérieures (CAIM) témoignent des nouvelles stratégies identitaires au profit de l'architecte d'intérieur, créateur de modèles ou plus tardivement designer. La spécialisation et l'élargissement de compétences du décorateur au cours de cette période révèlent des porosités et des différenciations professionnelles toujours difficiles à légitimer.

MOTS CLÉS: Décorateur. Artiste décorateur. Ensemblier. Architecte d'intérieur. Arts décoratifs. Professionnalisation.
1. Cet article reprend les idées principales d'une communication présentée au colloque international organisé par 1'Institut national d'histoire de l'art (INHA) le 07-08 octobre 2016 intitulé Pour une bistoire culturelle $d u$ décorateur, de la fin du XVIIIe à la fin $d u \mathrm{XXe}$ siècle.

2. Doctorante en Histoire de l'art, sous la direction de Monsieur le professeur Rémi Labrusse, Université Paris Nanterre, Ecole doctorale Milieux, Cultures et Sociétés du Présent et du Passé (MCSPP), Unité de recherches (EA 4414 HAR), enseignante en histoire de l'art à l'Académie Charpentier, Ecole d'Arts Appliqués (Paris). E-mail : $<$ beatrixgrondin@yahoo.fr> 
RESUMO: A evolução semântica da palavra "decorador" revela os processos identitários em jogo na construção das figuras de artistas. No alvorecer do século XX na França, os novos valores desse termo têm origem na afirmação do estatuto de artista criador e na consagração da síntese das artes. Uma figura ideal de artista decorador ganha forma, sem, contudo, referir-se a uma categoria profissional estável. Com efeito, não há consenso, no âmbito da recepção crítica, quanto às competências entre conhecimento e savoirfaire, colaboração independente e coordenação de projeto, visão estética e desenho técnico, concepção sob medida ou em série. Do ensemblier ao arquiteto de interiores, o termo "decorador" não adquire nenhuma definição social e profissional específica. A concepção da decoração de estilo francês moderna e a necessidade de responder às reais demandas da sociedade dividem os criadores. Os princípios racionalistas de arranjo dos espaços, defendidos pela Union des Artistes Modernes (UMA) em 1929, colocam definitivamente em crise a identidade do decorador ornamentista. A depreciação semântica que se prolonga durante os Trente Glorieuses culmina em reivindicações profissionais que giram em torno da figura do "arquiteto especializado". Associações como a Union des Artistes Décorateurs Créateurs d'Ensembles (UADCE) e sindicatos como o dos Créateurs d'Architectures Intérieures et de Modèles (CAIM) expressam as novas estratégias identitárias em favor do arquiteto de interiores, criador de modelos ou, mais tardiamente, designer. A especialização e a ampliação das competências do decorador durante esse período revelam porosidades e diferenciações profissionais que são sempre difíceis de legitimar.

PALAVRAS-CHAVE: Decorador. Artista decorador. Ensemblier. Arquiteto de interiores. Artes decorativas. Profissionalização.

ABSTRACT: The semantic evolution of the word decorator reveals the identity processes at stake in the construction of the image of artists. At the dawn of the 20th century in France, the new values of this word were originated in the affirmation of the status of creative artist and in the consecration of the synthesis of arts. An ideal image of a decorating artist takes shape, but does not, however, refer to a stable professional category. In fact, there is no consensus in the scope of critical reception with regard to the competencies, between knowledge and savoir-fare, independent collaboration and project coordination, aesthetic vision and technical design, tailor-made or serial designs. From ensemblier to interior architects, the term decorator does not acquire any specific social and professional definition. The concept of modern French style decoration and the need to respond to real demands from society divides creators. Rationalist principles of arrangement of spaces, defended by the Union des artistes modernes (UMA) in 1929, placed the identity of ornamental decorators in definitive crisis. The semantic depreciation that extends during the Trente Glorieuses culminates in professional claims that revolve around the idea of the "trained architect". Associations such as the Union des artistes décorateurs créateurs d'ensembles (UADCE) and unions such as the Créateurs d'architectures intérieures et de modèles (CAIM) represent new identity strategies in favor of the interior architect, model maker or, later, designer. The specialization and expansion of decorator's skills during this period reveals the porosity and professional differences that are always difficult to legitimize.

KEYWORDS: Decorator. Decorator Artist. Ensemblier. Interior Architect. Decorative Arts. Professionalization. 
Les désignations professionnelles d'artistes témoignent des mutations de pratiques et des enjeux de reconnaissance sociale. Pour interroger les figures du décorateur, le regard de l'observateur peut se focaliser sur les stratégies d'autodéfinition des groupements indépendants, Sociétés ${ }^{3}$ ou syndicats ${ }^{4}$ et sur la réception sociale qui en est faite. Les fluctuations sémantiques révèlent toute la tension des constructions identitaires à l'œuvre.

Au XIXe siècle, le vocable "décorateur" désigne en France un grand nombre de pratiques. Fleuristes ou architectes utilisent l'adjectif indiquant la spécialisation décorative de leur profession. Dans le domaine de la décoration intérieure et domestique, le terme "ensemblier" s'impose pendant les années 1910. Mais il ne trouve pas sa définition sociale et professionnelle propre. Les critiques se divisent sur les questions de savoir et savoir-faire, collaboration indépendante et maîtrise d'oeuvre, vision esthétique et dessin technique, conception sur mesure et en série. L'indétermination de compétences et de pratiques se renforce lors de la scission de la Société des artistes décorateurs (SAD) et de l'Union des artistes modernes (UAM) en 1929. Ce groupe rejette le terme "décorateur" dans sa dénomination officielle tout comme les créateurs de modèles, architectes d'intérieur, architectes designers des Trente Glorieuses. Les processus de conception globale, relevant de l'art, de l'artisanat et de l'industrie, aboutissent à de nouvelles différenciations et porosités professionnelles. Néanmoins, les diverses modalités de reconnaissance ne parviennent pas à dissiper la confusion sur leurs spécificités identitaires. Eł le décorateur ne semble plus incarner la figure artistique légitime pour réformer le cadre moderne de la vie quotidienne.

À partir de l'instabilité sémantique du vocable "décorateur", il sera donc possible de montrer la difficulté d'identification des pratiques professionnelles des concepteurs d'espace et d'objets. Dans cette optique, l'examen des documents de Sociétés et syndicats, les correspondances entre pouvoirs publics et associations, la documentation professionnelle des décorateurs tout comme le dépouillement de revues spécialisées offrent des matériaux d'analyse pertinents. Pour cet article, le corpus comprend plus précisément : le fond d'archives de la SAD ${ }^{5}$, des catalogues de Salons, une partie des Archives nationales de la Direction de l'Architecture consacrée à la profession de l'architecteo (1960-1970) ou encore le fond Jannette Laverrière.?

Ce matériel de recherches permet de postuler que les principes rationalistes de l'aménagement moderne mettent l'identité professionnelle du décorateur français en crise. Dans un premier temps, nous analyserons la figure du décorateur à partir des terminologies "artiste décorateur" et "ensemblier" au cours de la première moitié du XXe siècle. Puis, nous nous attacherons aux nouvelles définitions engagées pendant les Trente Glorieuses, par les groupements professionnels de l'Union des artistes créateurs d'ensembles (UADCE) et du syndicat des créateurs d'architectures intérieures et de modèles (CAIM).
3. Voir la bibliographie en fin d'article.

4. Il faut signaler le projet de recherches Labex CAP, 2015/2016 « De l'art à la politique, les artistes et le syndicalisme intellectuel dans l'entre-deux-guerres, le cas de la Confédération des Travailleurs Intellectuels (CTI). "

5. Archives de la Société des Artistes Décorateurs, Bibliothèque des Arts Décoratifs (MAD), Paris.

6. Les dossiers relatifs aux relations architectes et architectes d'intérieur regroupent des demandes relatives à la reconnaissance de la profession auprès des pouvoirs publics, à l'établissement d'un barème d'honoraires, à la création d'un Ordre, ainsi que des rapports de synthèse et notes de 1967-1969 (Archives nacionales Association des Créateurs de Modèles en Série/CAIM 19930521/86).

7. Fonds Janette Laverrière, $P \quad 61 C$, Bibliothèque Kandinsky, Centre de documentation et de recherche du Musée national d'art moderneCentre de création industrielle, Paris. 
8. Cf. Magnusson (2009).

9. Blondel (1777).

10. Cf. D'Aviler (1755). Selon Quatremère de Quincy (1832), le décorateur peut être peintre, sculpteur, architecte.

11. Nouveau ... (1856, p.147).

12. Dictionnaire... (18731874, p.992).

13. Cf. Bracquemond (1885).

14. La décoration "désigne aussi bien le produit d'une profession spéciale qu'une certaine qualité contenue dans des œuvres qui peutêtre n'ont jamais eu de destination décorative. De là, deux signification bien distinctes." (Bracquemond, 1885, p. 193). Désormais il existe une production artistique indépendante, sans destination précise "qui peut renfermer toute l'étendue d'art que la décoration comportait primitivement. De là, des formules, des pratiques, des recettes, dont le décorateur de profession semble plus que les autres artistes avoir conservé la tradition. De là, aussi, l'apparence d'un art particulier. " (Bracquemond, 1885, p.198-199).

15. Havard (2012, p.45-46).

16. Havard (2012 p. 46).

\section{L'ÂGE D'OR DU DÉCORATEUR FRANÇAIS}

\section{Un artiste spécialisé?}

Rappelons qu'au XVIIle siècle, le "décorateur" ne désigne pas encore un professionnel spécialisé de l'habitation et de l'aménagement intérieur. Bien qu'il existe dans les pratiques ${ }^{8}$, l'usage du terme varie selon les discours sur l'art. Dans son Cours d'architecture publié en 1777, le théoricien Jean-François Blondel ${ }^{9}$ considère la décoration comme la branche essentielle de l'architecture. Le terme glorifie les hautes qualités des grands maîtres de l'Art, peintres, sculpteurs ou architectes. ${ }^{10}$ Néanmoins, les jugements esthétiques sur les excès décoratifs déprécient déjà ce vocable.

Le dictionnaire Larousse en $1856^{11}$ définit le décorateur de manière plus restrictive comme un "professionnel de l'ornementation". Puis le Littré précise "celui dont la profession est d'orner l'intérieur des appartements ou celui qui fait des décorations pour les théâtres, pour les fêtes ".$^{12}$ Les nombreux manuels professionnels du XIXe siècle attestent de la vulgarisation de l'adjectif, employé indistinctement pour qualifier un architecte, un tapissier, un ébéniste ou un pâtissier (Figure 1). Des glissements sémantiques s'observent dans la dilution du vocable. L'adjectif précisera désormais la finalité décorative d'un métier ${ }^{13}$ ou d'un objet, indépendamment de sa qualité esthétique. Parallèlement, le terme se généralise sous sa forme nominale pour distinguer une catégorie professionnelle dans le champ artistique de la décoration intérieure. Selon l'artiste Félix Bracquemond, cette profession n'aurait que «les apparences d'un art particulier »..$^{14}$ Or, dans le Dictionnaire de l'ameublement et de la décoration de 1894, l'historien de l'art Henri Harvard identifie une spécialisation: "On donne le titre de décorateur aux artistes qui s'occupent plus spécialement de la décoration intérieure ou extérieure des édifices ». ${ }^{15}$ Tout en déplorant un déclin de compétences, l'auteur s'étonne de ce récent changement lexical et professionnel au regard de la tradition artistique et de la hiérarchie des arts.

La différence qu'on fait aujourd'hui, comme profession, entre les artistes peintres et sculpteurs proprement dits et leurs confrères portant le nom de décorateurs aurait été considérée, dans les siècles précédents, comme parfaitement ridicule. Les plus grands artistes, au XIVe et XVe siècle, ne regardaient pas comme en dessous d'eux de se charger de travaux de pure décoration. ${ }^{16}$ 


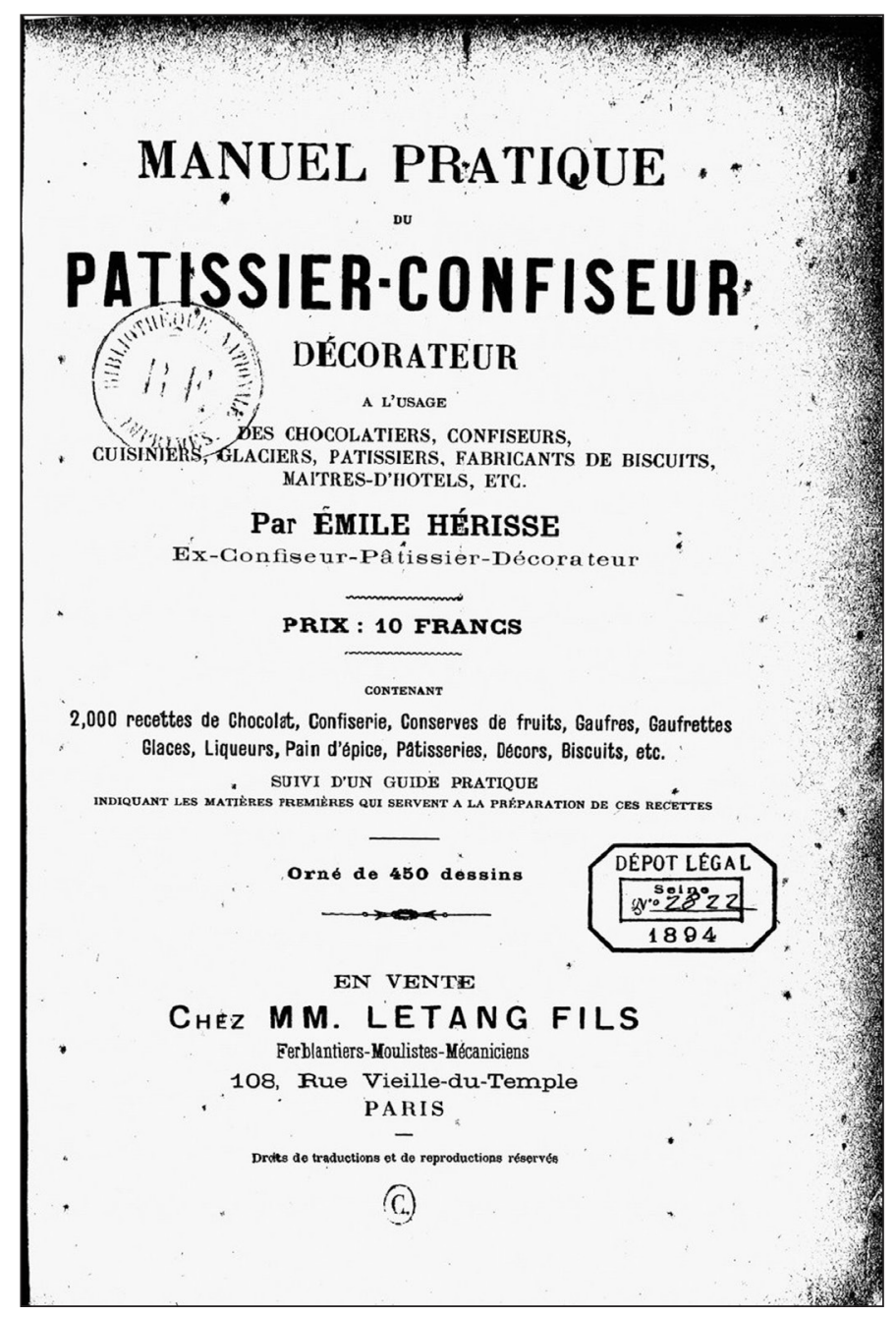

Figure 1 - Couverture du Manuel Pratique du Pâtissier -Confiseur Décorateur : à l'usage des chocolatiers, confiseurs, cuisiniers, glaciers. Source: Bibliothèque Nationale de France

La question de la spécialisation est à mettre en relation avec l'abolition des corporations en 1791. D'autres facteurs agissent comme la nouvelle parcellisation du travail, la crise de l'apprentissage et plus généralement, l'avènement d'un nouveau système de production de biens de consommation imposé par l'industrie tout au long du XIXe siècle. L'organisation des métiers d'art s'en trouve fortement bouleversée et les arts décoratifs réévalués. Un mouvement réformiste en faveur des "arts utiles"17 s'engage. Citons sans exhaustivité l'importance des expositions comme celles de l'Union centrale des arts décoratifs en 1882 et 1883, I'Exposition universelle de 1889, ou encore les Salons organisés au palais des ChampsElysées ouverts aux arts dits mineurs. En 1893, la Société Nationale des Beaux- 
18. Les galeries parisiennes multiplient aussi les expositions d'arts décoratifs comme les magasins de l'Art nouveau de Siegfried Bing en 1895 et la Maison Moderne de Julius MeierGraefe ouverte en 1895.

19. Cf. Froissart-Pezone (2004); Meneux (2006).

20. Cf. Froissart-Pezone (2004, p. 208).
Arts admet non seulement les objets d'art isolés mais aussi l'ameublement et la décoration intérieure. ${ }^{18}$ À partir de 1903, le Salon d'automne propose une section particulière. Eł en 1904, année de l'inauguration du Musée des arts décoratifs à Paris, la SAD présente son premier Salon exclusivement dédié aux arts décoratifs (Figure 2).

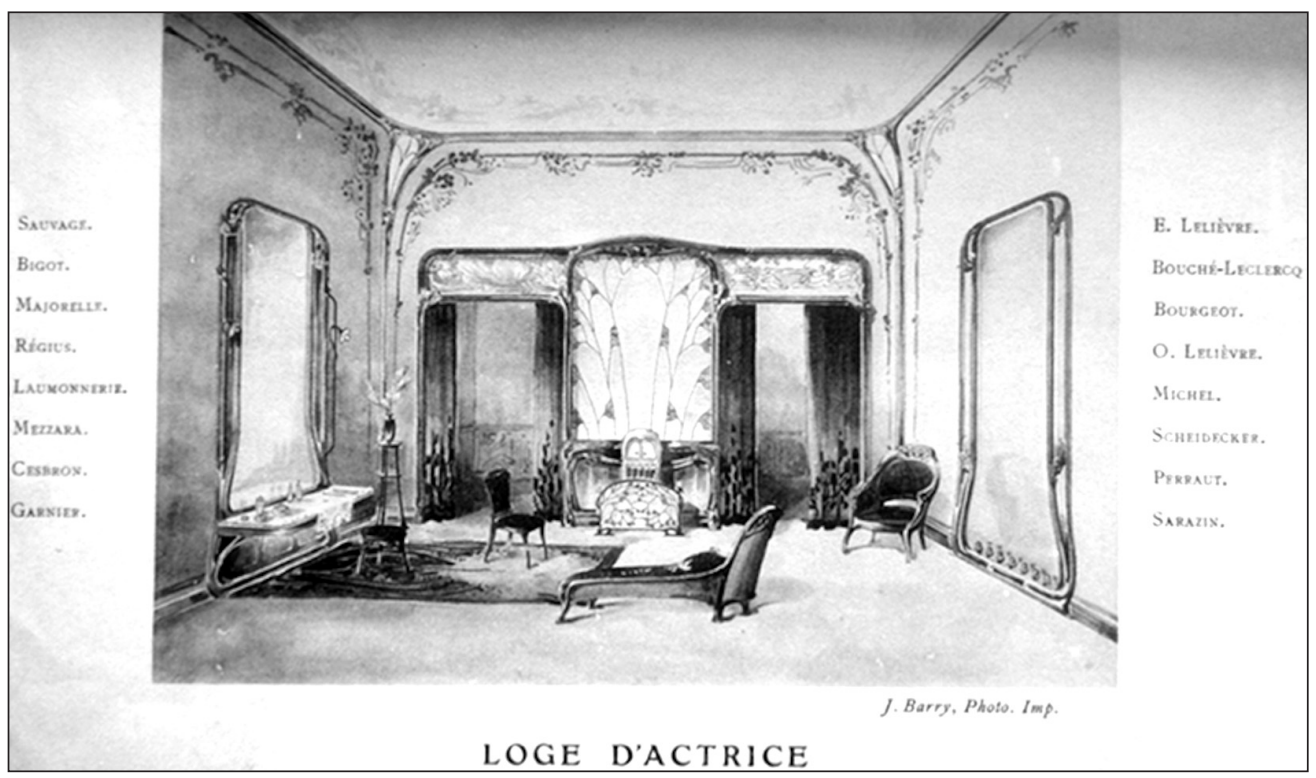

Figure 2 - Henri Sauvage, Charles Sarazin (et collaborateurs), ensemble "Loge d'actrice" présenté au premier Salon de la Société des artistes décorateurs (janvier-février 1904) au Petit Palais de Paris. Source : Société des artistes décorateurs (1904).

Si l'idéal de l'unité des arts et de "I'Art dans tout", 19 partagé par de nombreux artistes européens, permet de comprendre l'enjeu des collaborations artistiques, une Société française, comme celle des artistes décorateurs, répond en priorité à des revendications professionnelles et corporatistes. Pour résoudre le malaise esthétique et économique révélé à l'Exposition universelle de 1900, des stratégies "séparatistes" de reconnaissance voient le jour. L'historienne de l'art Rossella Froissart remarque très justement ce paradoxe : «(...) la professionnalisation de la figure de l'artiste décorateur mit fin à l'utopie de l'unité de l'art chère aux tenants de l'art social et au nom de laquelle les arts mineurs avaient acquis le statut d'art tout court.»20 


\section{La reconnaissance de l'artiste décorateur}

En 1901, la SAD ${ }^{21}$ consacre une terminologie valorisante pour tous les métiers d'arts décoratifs. Sans rendre compte de l'hétérogénéité des profils sociaux, elle englobe une diversité de pratiques (reliure, orfèvrerie, verrerie, céramique, broderie, tapisserie, sculpture, mobilier ou peinture décorative par exemple). Dans l'annuaire du premier catalogue de 1904, des artistes se désignent toujours par leur technique artisanale (émailleur, céramiste, ferronnier d'art). D'autres choisissent l'expression "artiste décorateur", plus honorifique ${ }^{22}$ et implicitement pluridisciplinaire. ${ }^{23}$ De manière performative, ce titre général réunit idéalement, sans précision de métier ou de branche décorative: I'artiste et le décorateur, l'artiste et l'artisan, le créateur ${ }^{24}$ et l'ouvrier. ${ }^{25}$ Par ailleurs, les statuts de la SAD ne distinguent pas le mode de production (artisanal ou industriel) à condition que l'œuvre ne soit pas une copie de style. Mais dans les pratiques des sociétaires ou exposants, si le pastiche s'éclipse, l'art industriel de grande série n'est pas privilégié. La reconnaissance du statut préoccupe davantage le Comité de la Société qui se mobilise pour le projet d'amendement de la loi des 19-24 juillet 1793 en faveur des dessinateurs et des sculpteurs. II sera promulgué le 10 mars 1902. La protection juridique apparaît comme un enjeu majeur qui précède l'organisation du Salon. L'avocat René Guilleré, membre fondateur de la SAD, hiérarchise ainsi les priorités:

La tâche est d'abord de faire reconnaître les artistes décorateurs, par les pouvoirs publics, des droits égaux à ceux qu'ils accordent aux artistes dits des beaux-arts, afin d'établir leur qualité d'artiste et de reconnaître leur importance sociale ; ensuite d'obtenir des lois, des jurisprudences et des pratiques commerciales, la protection de leurs intérêts artistiques ; enfin, d'organiser des expositions qui manifestent leur talent. ${ }^{26}$

Dans son discours aux accents ruskiniens, René Guilleré défend le renouvellement des arts décoratifs par la reconnaissance de l'artiste et son épanouissement personnel.

Pour que l'art soit prospère, il faut que l'artiste soit heureux (...) il ne suffit pas de songer d'aucuns appellent 'les nobles intérêts de l'art'. II faut nous préoccuper aussi de la situation morale et matérielle de l'artiste. L'artiste ne peut produire en perfection que s'il retire de son œuvre des satisfactions morales et des avantages matériels. ${ }^{27}$

La reconnaissance sociale ne se comprend pas sans le leitmotiv du nationalisme ${ }^{28}$ artistique et la lutte contre les concurrences étrangères menaçant les industries d'art. Prenant appui sur les rapports de Marius Vachon, ${ }^{29}$ I'ambition corporatiste $^{30}$ de la SAD s'affirme clairement. Toutefois, si tous les artistes peuvent être décorateurs et si tous les artistes décorateurs sont des décorateurs, ${ }^{31}$ le terme décorateur n'en demeure pas moins imprécis pour identifier ses compétences
21. La SAD est une association loi 1901 , reconnue d'utilité publique en 1924, fondée le 7 février 1901 par une quarantaine d'artistes ayant répondu à l'appel lancé en 1900 par l'avocat de l'Association des sculpteurs modeleurs, René Guilleré, président de la SAD en 1911

22. Sur l'ensemble des sociétaires de 1904, soit environ 300 membres, seulement Charles Fridrich se désigne décorateur et Lucien Ott architecte décorateur.

23. Par exemple, AlbertErnest Carrier-Belleuse (1840-1917), formé à l'Ecole des Beaux-Arts avant d'ouvrir son propre atelier de sculpteur-ornemaniste, est dans sa pratique professionnelle surtout sculpteur, alors que des artistes comme Maurice Dufrêne seront davantage pluridisciplinaires concevant tout autant des bijoux que des meubles.

24. Eugène Gaillard souhaite, en 1911, l'ajout d'un sous-titre à la SAD : "Artistes créateurs des décors et des modèles prototypes de nos arts appliqués modernes".

25. Les statuts de la Société, publiés en 1904, admettent pour membres actifs des "artistes ayant fait personnellement œuvre d'art", des "créateurs de modèles d'art appliqué ou d'œuvres ayant une destination décorative d ét e r m i n é e (Bulletin...1902, p. 18)

26. Bulletin... (1902, p. 22). 27. Bulletin... (1902, p. 38). 28. Cf. Froissart-Pezone (2005).

29. Vachon (1886, 1899).

30. La question de la 
corporation est d'actualité. (Anonyme, 1902).

31. “(...) D'ailleurs le même artiste n'est-il pas tout à tour statuaire et décorateur? " (Société des artistes décorateurs, 1901, p. 45).

32. La question de la spécialisation se retrouve aussi dans le syntagme "art décoratif". " (..) l'art appliqué aux objets usuels, pour ne pas employer le mot art décoratif qui ne spécialise rien (...) ". (Boisseau,1912, p.18).

33. Belville (1910, p. 18-19).

34. Belville (1910, p. 18).

35. Pendant la première moitié du XXe siècle, les formations artistiques et techniques sont dispensées à l'École supérieure des Arts décoratifs ouverte depuis 1767 (Paul Follot, André Mare, René Gabriel), dans les Écoles régionales de Beaux-arts et des arts appliqués (comme celle de Nancy), à l'École Boulle (Armand-Albert Rateau) qui assure outre ses cours du soir, des formations spécialisées aux métiers d'art dès le plus jeune âge (ébénistes, menuisiers en sièges, tapissiers, sculpteurs sur bois, ciseleurs, monteurs en bronze, graveurs etc.). Certains créateurs sortent diplômés de l'École nationale supérieure des Beaux-arts (par exemple Paul Iribe et Pierre Chareau). La Chambre Syndicale de l'Ameublement délivre également des cours, tout comme l'ancien Comité des dames, fondé en 1895 par l'UCAD, devenu " École et ateliers d'art décoratif" vers 1909, dirigé par René Prou en 1928 puis mobilisé pour la création du Centre d'Art et de techniques (future école Camondo) fondé en 1944 qui délivrera un diplôme de décorateurensemblier. professionnelles. ${ }^{32}$ Dans un article de janvier 1910, l'artiste Eugène Belville souligne la confusion régnant dans la réception sociale:

Ce qualificatif lui-même (décorateur), d'ailleurs parfaitement impropre, commence à peine à être compris pour désigner les artistes voués aux arts dits appliqués, industriels, décoratifs ou mineurs. Pour beaucoup de gens de bonne éducation, le décorateur ne peut être qu'un peintre ; artiste, s'il fait des décors de théâtre ; ouvrier, s'il transforme en marbre ou bois précieux le sapin des devantures de boutiques. Le décorateur créateur de formes et d'harmonies de lignes ou de couleurs s'appliquant à tous les 'arts de la vie' $(\ldots) .^{33}$

L'auteur rappelle que la création de la section "objets d'art" de la Société Nationale des Beaux-Arts « suscita chez beaucoup de décorateurs qui se contentaient jusque-là d'abandonner leur modèles à l'exécutant l'envie de prendre eux-mêmes la responsabilité de la réalisation de leurs projets ». ${ }^{34}$ Pour l'artiste rattaché à une entreprise industrielle, se pose le problème du droit à la signature, quant à d'autres profils observés par l'auteur, se distinguent les créateurs spécialisés par leur formation mais pluridisciplinaires dans leurs pratiques (comme le fut par exemple Henry BelleryDesfontaines (1867-1909), l'un des membres fondateurs de la SAD) (Figure 3).

Concepteurs-exécutants, indépendants ou employés, artistes créateurs de modèles spécialisés ou non, de formation et pratiques diverses, ${ }^{35}$ le flou entourant le terme "décorateur" ne facilite pas la reconnaissance sociale de ce qu'il est, de ce qu'il produit et comment il le produit. Or, institutionnaliser une profession nécessite une légitimité sociale.

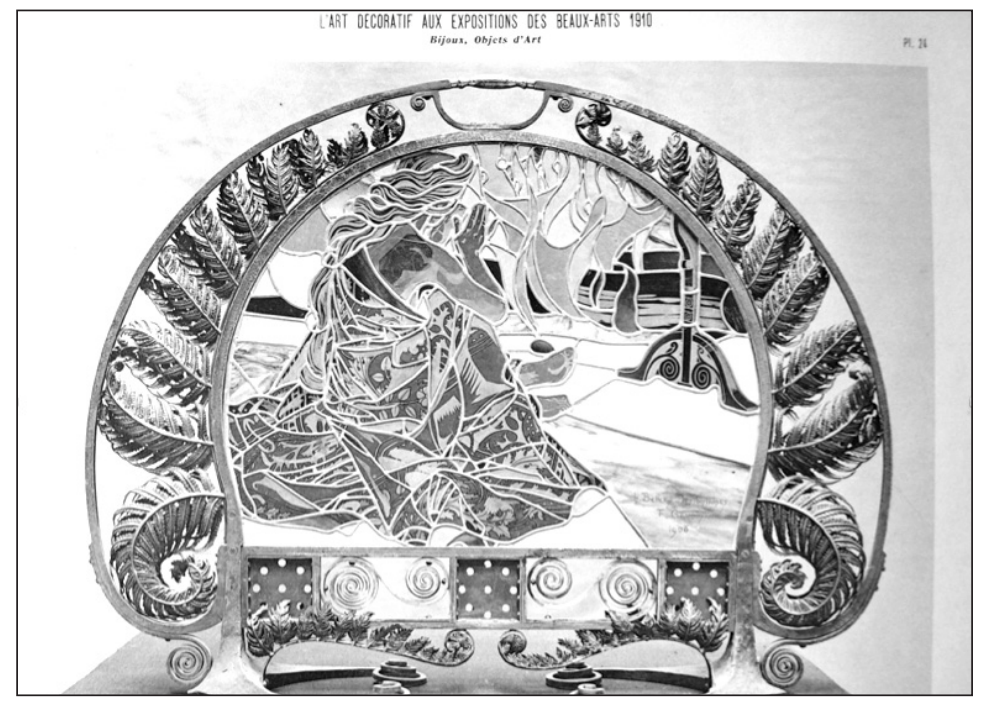

Figure 3 - Exposition rétrospective de Henry Bellery-Desfontaine au Salon des artistes décorateurs de 1910, Paris. Source : Guérinet (1910). 
Le décorateur comme ensemblier

Au cours des premières décennies du XXe siècle, la consécration de I'"ensemble" moderne induit le terme "décorateur", non adjectivé, à se cristalliser autour d'une figure qui coordonne tous les arts du décor intérieur. ${ }^{36}$ La presse de l'époque reconnaît l'impulsion déterminante donnée par les Allemands à l'Exposition universelle de 1900, puis au Salon d'Automne de 1910.37 C'est par l'ensemble que l'unité stylistique moderne française doit se réaliser. Le sculpteur Maurice Maignan en appelle à l'harmonisation artistique générale (Figure 4).

Et nous entrevoyons un avenir où l'art ne fabriquera plus des objets d'art-fussent-ils dénommés chefs-d'œeuvre-, mais où il produira de l'harmonisation. (...). Notre art décoratif doit chercher à s'imposer comme l'art des ensembles. II doit devenir l'art des arts et commander à toutes les facultés artistiques, fussent-elles des plus grands peintres ou des plus grands sculpteurs. Et c'est pourquoi le plan de son organisation est nécessaire, ce plan cherchant à disposer, à mettre en scène, les talents les plus divers. ${ }^{38}$

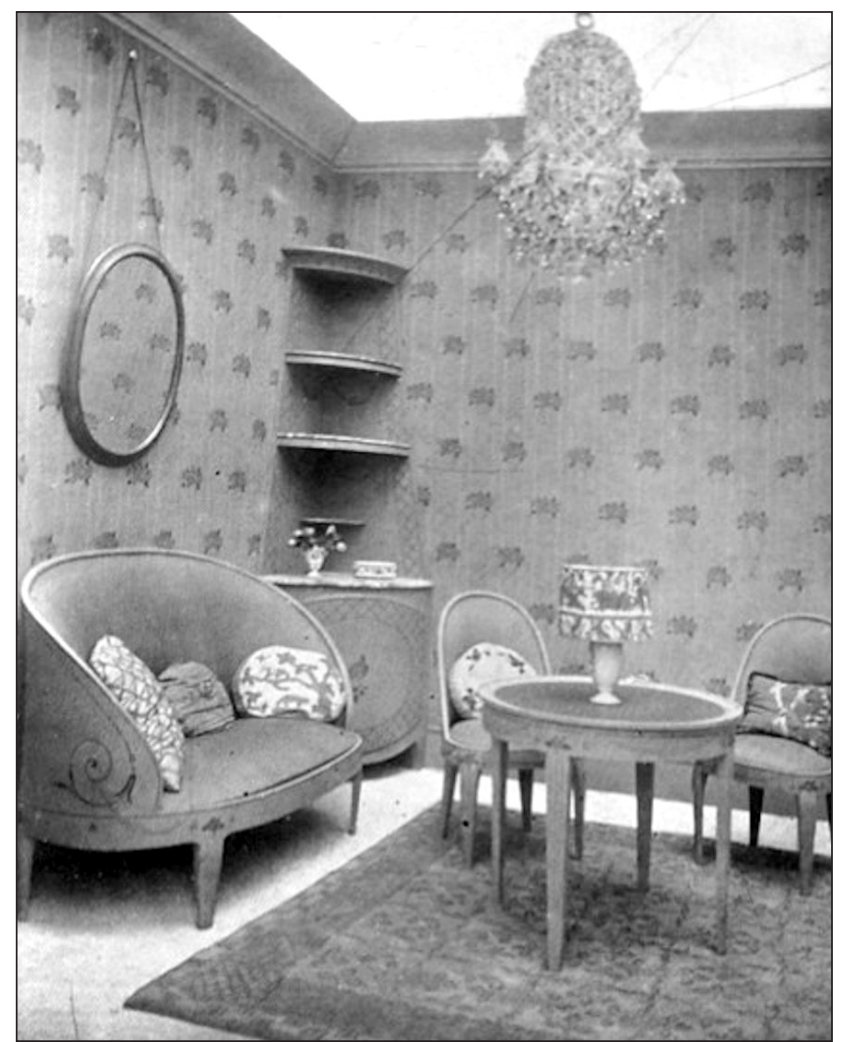

Figure 4 - Ensemble français de Louis Süe et Paul Huillard présenté Salon d'Automne de 1910, Paris. Source: Vauxcelles (1910, p. 167).
36. Cf. Genuys (1904).

37. Cf. Vauxcelles (1912).

38. Maignan (1911, p. 201202). 
39. Le Larousse universel en 1922 définit l'ensemblier comme l'artiste qui combine l'ensemble de la décoration. L'ensemble est le "cadre charmant qui donne l'illusion de la vie, la sensation d'un intérieur habité, qui pique surtout l'attention. Nos décorateurs, la plupart hommes de goût et d'esprit ingénieux, s'en donnent à coeur joie. Aussi a-t-on créé depuis une quizaine d'année la fonction et le terme d'ensemblier." (Anonyme 1923, p. 1121).

40. Anonyme (1912, p.757).

41. Exposition... (1910).

42. Dans son premier bulletin de janvier 1902, la S A D d é p lor e l'individualisme artistique français: "Chacun reste isolé et travaille pour se satisfaire personnellement, préoccupé seulement d'épuiser sur son œuvre toute sa virtuosité d'homme de métier, mais sans respect de l'ensemble. Chacun joue un solo dans l'orchestre ". (Exposition...,1910, p.37).

43. "Tout, dans leurs ensembles décoratifs (des Munichois), se sent, vaut par de solides mérites de cohésion, de solidarité, de subordination des exécutants à la pensée directrice du 'maître d'œuvre'. Tout, chez eux, est conçu et réalisé en vue de l'effet d'ensemble; les moindres moulures ont été dessinées par l'architectechef. Chez nous, (...) L'accord ne règne pas toujours entre les collaborateurs ; chacun tire sa couverture à soi ; chacun veut 'chanter son grand air'. Nous n'avons guère le respect du chef d'orchestre, il faut l'avouer. " (Anonyme, 1910, p. 455).

44. Roches (1911, p. 317).

45. Charles Hairon dans le catalogue du Salon de 1930
L'ensemble décoratif place ainsi le décorateur face à des enjeux artistiques et économiques majeurs. Pour les critiques défenseurs d'un art social, ce rôle ne fait pas de l'ensemblier ${ }^{39}$ une figure futile comme celle dépeinte en 1912 dans l'hebdomadaire La vie parisienne.

Un ensemblier est un monsieur qui, sous prétexte de rénover le goût présent- se portait-il si malẹ- entend montrer à ses contemporains comment un artiste peut orner sa demeure. Allez au Salon, vous verrez comme c'est facile. On met un coussin jaune d'œuf sur un tapis épiscopal, des tentures vert pomme à côté de murs indigo, des meubles peints en rose avec des soieries noires... Et voilà un 'ensemble' ! Abstenons-nous de juger. II ne faut faire aux ensembliers nulle peine, même légère. L'autre jour, devant leur exposition, quelqu'un exprimait sans ambage une opinion sans indulgence, alors une petite femme, haussant les épaules:

Vous ne voyez donc pas que c'est stylisé?

Pauvre petite femme !....Pauvre nous surtout car, je vous le demande, qu'allons-nous devenir cet hiver si nous sommes reçus par des ensemblières? 40

Dans son rapport sur la section française de l'Exposition internationale de Milan en 1906, l'architecte et décorateur Eugène Pierre Selmersheim distingue deux figures idéales et complémentaires à partir des ensembles présentés par l'artiste Maurice Dufrêne et l'architecte Louis Bigaux : La figure de l'artiste isolé et le maître d'œuvre coordinateur. L'un (le premier) représente le créateur qui conçoit et exécute tout lui-même, l'autre figure (le second) conçoit mais travaille avec des collaborateurs, des artistes décorateurs et des industriels. Pour réformer les arts décoratifs, I'un devrait être l'exception et l'autre "la majorité". ${ }^{41}$ Soutenu par des revues spécialisées comme Art et Décoration, Art et Industrie ou encore L'Art décoratif, l'ensemble sera progressivement promu par les Salons et les expositions internationales. Les collaborations coordonnées se mettent difficilement en place, ${ }^{42}$ la faute incombe aux artistes jugés trop individualistes. ${ }^{43}$ L'écrivain Fernand Roches s'interroge:

A quand la volonté qui saura organiser ces talents de décorateurs, les diriger, les éclairer, afin que, au lieu de s'émietter, ils concourent dans la subordination, à l'avènement d'une expression décorative, traditionnelle, logique, utile et nécessaire? ${ }^{44}$

Le même constat des années 1910 ressort lors de la confrontation du Deutscher Werkbund au Salon des artistes décorateurs en 1930.45 Contrairement aux Allemands, I'harmonie stylistique des ensembles français résulte d'une référence 
commune à la grande tradition nationale et des affinités artistiques ${ }^{46}$ (Figure 5). Les initiatives entreprises ne bouleversent pas l'organisation entre les divers corps de métiers et l'industrie. À la différence de la discipline allemande, une figure d'autorité ne s'impose pas en France. La présentation de la Maison cubiste au Salon d'Automne de $1912^{47}$ annonce une nouvelle ère autour de l'oeuvre collective. De l'atelier Martine à la Compagnie des arts français, bien que I'alliance privilégiée soit celle de l'art et de l'artisanat de luxe, la décoration intérieure moderne doit se concevoir en collaboration. Elle requiert une figure ordonnatrice moins "démiurgique" que celle apparve pendant l'Art nouveau, distincte de "l'ensemblier huchier","auteur" ou "essayiste en quête de style" selon le critique d'art Guillaume Janneau. ${ }^{48}$

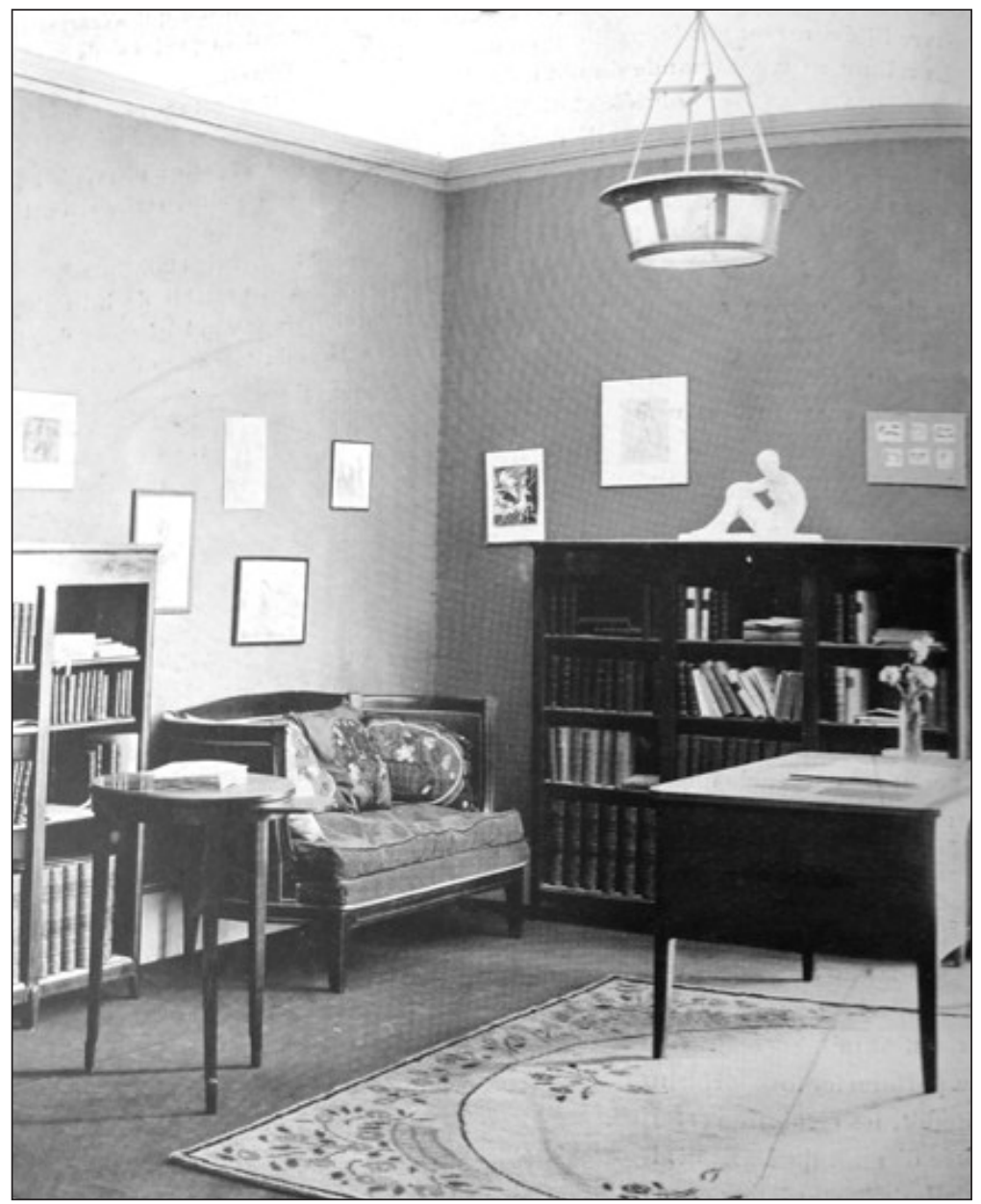

Figure 5 - Paul Follot, chambre à coucher, Salon de la Société des artistes décorateurs, 1923, Paris. Source: Archives Société des artistes décorateurs, Bibliothèque des arts décoratifs, Paris le souligne : "l'animateur de la section allemande, en fixant le thème spécialement choisi par lui, a imposé une discipline à tous ses collaborateurs, artistes, producteurs, commerçants, unis sous sa direction pour servir un idéal national. (...) Notre Société comprend, en effet, des créateurs de toute tendance, désireux d'affirmer, autant que possible leur individualité ", non paginé.

46. Cf. Fravalo (2012).

47. Cf. Possemé (1999).

48. Janneau (1925). 
49. Anonyme (1912, p. 157).

50. Maurice Maignan date ce changement terminologique dès 1905 lorsque les meubliers Art nouveau sont devenus ensembliers «Vers 1905 son ambition (celle du décorateur) réussit à aborder de grandes surfaces ; il prend le nom d'ensemblier, oui, il va faire des ensembles. (...) Vers 1910, le voici construisant un hôtel particulier ". (Maignan, 1911, p. 195).

51. Cf. Clouzot (1925, p.349).

52. L'exemple de Paul Follot (1877-1941) est révélateur. Fils du fabricant de papiers peints Felix Follot, élève d'Eugène Grasset, il commence sa carrière en 1901 comme dessinateur pour la Maison moderne de Julius MeierGraefe et expose d'abord des bijoux vers 1905 avant de concevoir des meubles et des ensembles.

53. "L'architecture et l'art du tapissier, ce que l'on appelle aujourd'hui l'ensemblier (...) ». (Jaloux, 1934, p. 461).

54. Les critiques comme Louis Vauxcelles dans les commentaires du Salon d'Automne des années 1910 et 1911 ne cessent de rappeler que des peintres comme Gustave Jaulmes, Louis Süe et André Mare, représentants coloristes des années 1910, se sont improvisés meubliers (Vauxcelles, 1911).

55. "La section des 'ensembliers' comme on dit aujourd'hui pour désigner les tapissiers et les artistes du meuble (...) ". (Gillet,1921, p. 315).

56. "Trop de tapissiers (...) trop d'ébénistes ou trop de littérateurs parmi les
Le terme ensemblier se diffuse, voire se substitue à celui de "décorateur". Dans un article anonyme du 19 octobre 1912 de La vie parisienne: "L'ensemblier. C'est un mot nouveau (...) car on ne dit plus d'un artiste qu'il est décorateur mais qu'il est ensemblier (...) ».49 Au cours des années 1910 et bien avant son entrée dans les dictionnaires en 1922, ce néologisme inélégant s'utilise plus fréquemment dans la presse et les publicités professionnelles. ${ }^{50} \|$ reçoit son sacre ultime à I'Exposition internationale des arts décoratifs et industriels modernes de 1925. L'ensemblier est "le créateur de nos foyers" qui "réunit le décor fixe au décor mobile". ${ }^{51} \mathrm{Si}$ le terme décorateur ne désigne pas une figure précise, il en va de même pour l'ensemblier. ${ }^{52}$ Tantôt architecte, ${ }^{53}$ tapissier, peintre ${ }^{54}$ (Figure 6), meublier, ${ }^{55}$ sculpteur, son profil oscille entre l'artiste total, ${ }^{56}$ |'homme de métier ${ }^{57}$ et le créateur de modèle. Guillaume Janneau, bien qu'il reconnaisse que l'ensemblier soit "le nom noble du tapissier", 58 le considère avant tout comme un "fournisseur d'idée" qui n'est plus un "homme de métier". La même année, l'artiste décorateur Maurice Dufrêne admet la séparation inévitable entre la conception et l'exécution. Dès 1914, Maurice Pillard-Verneuil préconise la séparation professionnelle des compétences de conception, de fabrication et de commercialisation des oeuvres. ${ }^{59}$ Enfin, l'architecte et historien de l'art Lucien Magne ${ }^{60}$ envisage une figure distincte de celle de l'ébéniste et de l'architecte pour désigner le créateur de modèles appliqués à l'industrie. Parce qu'il doit être capable de répondre à toutes les réalités sociales, l'ensemblier ne peut se limiter au rôle de "tailleur sur mesure" au service de l'élite fortunée. Néanmoins, cette figure ne domine pas. Dans la filière de l'ameublement français, les liens privilégiés avec l'artisanat prévalent. L'ensemblier tient souvent sa propre boutique. Certains décorateurs travaillent avec de grands ateliers ou des maisons d'ameublement (comme les Maisons Gouffé ou Jansen par exemple). D'autres, comme Pierre Chareau, exerçent à l'intérieur d'un réseau relativement fermé de clients et d'artisans. Le critique d'art Henri Clouzot ne retient pas l'application à l'industrie comme compétence exclusive pour dessiner la figure archétypale du décorateurensemblier des années 1920. II insiste en revanche sur la fonction de "maître d'oeuvre" qui travaille à la commande, comme Jacques-Emile Ruhlmann, ArmandAlbert Rateau, Maxime Old, Jean Royère ou René Herbst par exemple.

L'ensemblier' conçoit, tout ce qui constitue 'l'ensemble' d'une pièce, soit qu'il en ait donné les dessins, soit qu'il les ait demandés à des collaborateurs travaillant sous sa direction, comme aux belles époques, les divers artisans groupés sous les ordres du Maître de l'oeuvre, puis de l'architecte. ${ }^{61}$ 


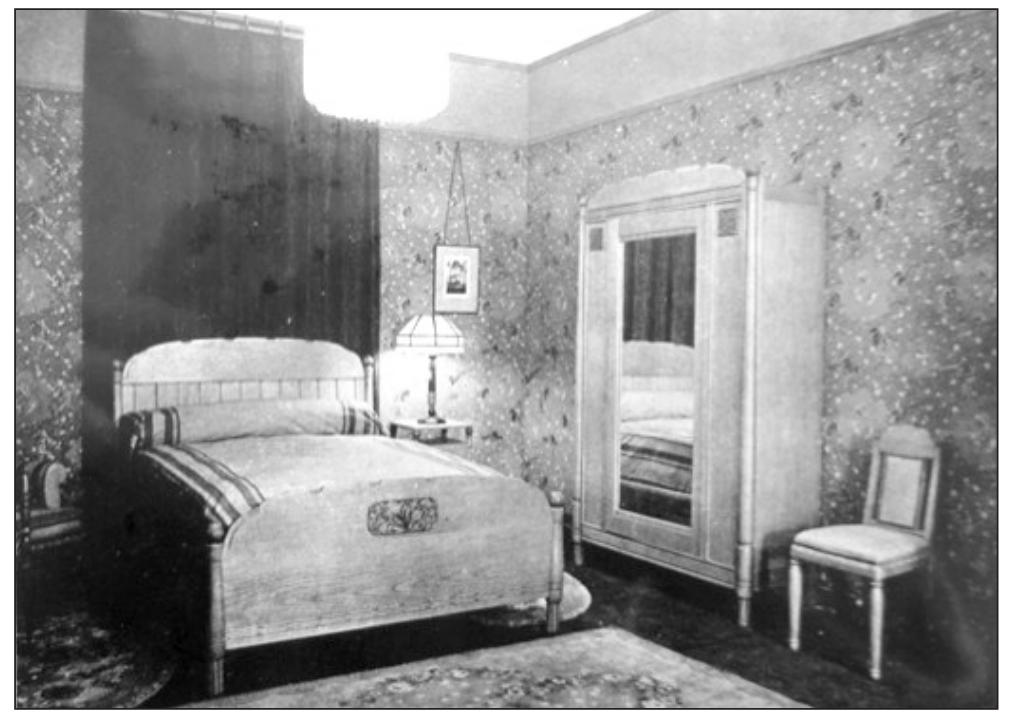

Figure 6 - André Mare, Cabinet de travail, Salon d'Automne 1912, Paris. Source: Roches (1912, p. 272).

\section{Un homme de goût}

Si le décorateur ensemblier nous apparaît comme une figure artistique polymorphe, la presse généraliste le décrit souvent comme esthète. Si les élites n'imposent plus leur goût, il incombe au décorateur de composer les styles, voire d'imposer le sien. ${ }^{62}$ Selon Pierre Albin, la maîtrise de la grammaire des styles s'avère indispensable.

La formation rapide des néo-capitalistes, due à l'exagération des bénéfices commerciaux, a donné naissance à une profession qui n'est pas nouvelle-il n'y a plus rien de nouveau sous le soleil, pas même les nouveaux riches-mais qui prend en ce moment une curieuse extension. Cette profession, à recommander aux jeunes gens en mal de trouver un débouché à leur activité, est celle des ensembliers. Un ensemblier est un monsieur ou une dame, le sexe peu importe, qui se charge, moyennant une équitable retribution, d'avoir du goût pour ceux à qui leur absence d'éducation individuelle ou de tradition familiale n'ont permis d'en acquérir. Sa fonction est d'assortir, ou comme les disent les clients des grands magasins, de nuancer. II joue en maestro avec la gamme de couleurs complémentaires. Le manuel de l'ameublement n'a pas de secret pour lui et il a L'art de reconnaître les styles: il sait quelles rayures caractérisent le Directoire et qu'il ne faut pas sur une pendule Empire, placer un Saxe Louis XV, mais quelque bronze romain ou égyptien. ${ }^{63}$

Quelque que soit le profil du décorateur, moderne ou antiquaire, "coloriste" ou "constructeur"64, la conception d'ensemble triomphe. décorateurs. Un décorateur doit être ébéniste, peintre architecte et, de plus, avoir une culture générale qui lui permette de bien situer son art, d'en connaître les possibilités et les bornes ". (Cogniat, 1935, p. 162).

57. " On avait imaginé, avant la guerre, pour designer l'artiste chargé de discipliner et de coordonner l'action de tous les corps de métier dans la décoration intérieure d'une maison, un mot aussi villain qu'inutile, l'ensemblier. (...) Ici, tous les corps de métiers sont réunis en un seul homme qui est à la fois l'inspirateur, l'ordonnateur et l'ouvrier. C'est lui qui a dessiné tous les détails (...) ». (Michel, 1914, p. 173).

58. Janneau (1925, p. 141)

59. Pillard-Verneuil (1914).

60. Magne (1913).

61. Clouzot (1925, p. 349).

62. En 1884, dans un manuel adressé aux classes moyennes supposées sans éducation artistique, le décorateur est en charge de l'aménagement intérieur, comme l'architecte et contrairement au tapissier. (Cardon, 1884).

63. Albin (1922, p. 1).

64. L'expression est utilisée par la plupart des critiques des années 1910 comme Guillaume Janneau par exemple qui distingue les "coloristes" André Groult, Louis Süe, Clément Mère, des "constructeurs" Léon Jallot, Maurice Dufrêne et Pierre Selmersheim. 
Le discrédit des Modernes

Dès 1929, les tenants de l'UAM déplacent le discours des besoins et des usages dans le contexte des urgences sociales de l'époque. Ils définissent le décorateur comme un spécialiste, non de l'ornementation au service d'une élite, mais de l'habitation et de l'équipement au service de la collectivité. Si l'ensemblier peut être vu comme la figure moderne qui incarne dans sa terminologie l'idéal de la synthèse des arts, c'est dans l'interprétation et les modalités de cette synthèse que la figure du décorateur finira par vaciller. L'UAM, qui se veut pluridisciplinaire, rejette la décoration d'embellisement, de l'accessoire et du mensonge. Dans les textes, alors que le vocable "ensemblier" résiste, celui de "décorateur" disparaît au profit de I'"architecte d'intérieur" (Figure 7). Tant dans ses dimensions esthétiques, ses finalités sociales que dans son champ d'applications, le décorateur moderne devra redéfinir ses pratiques et sa désignation.

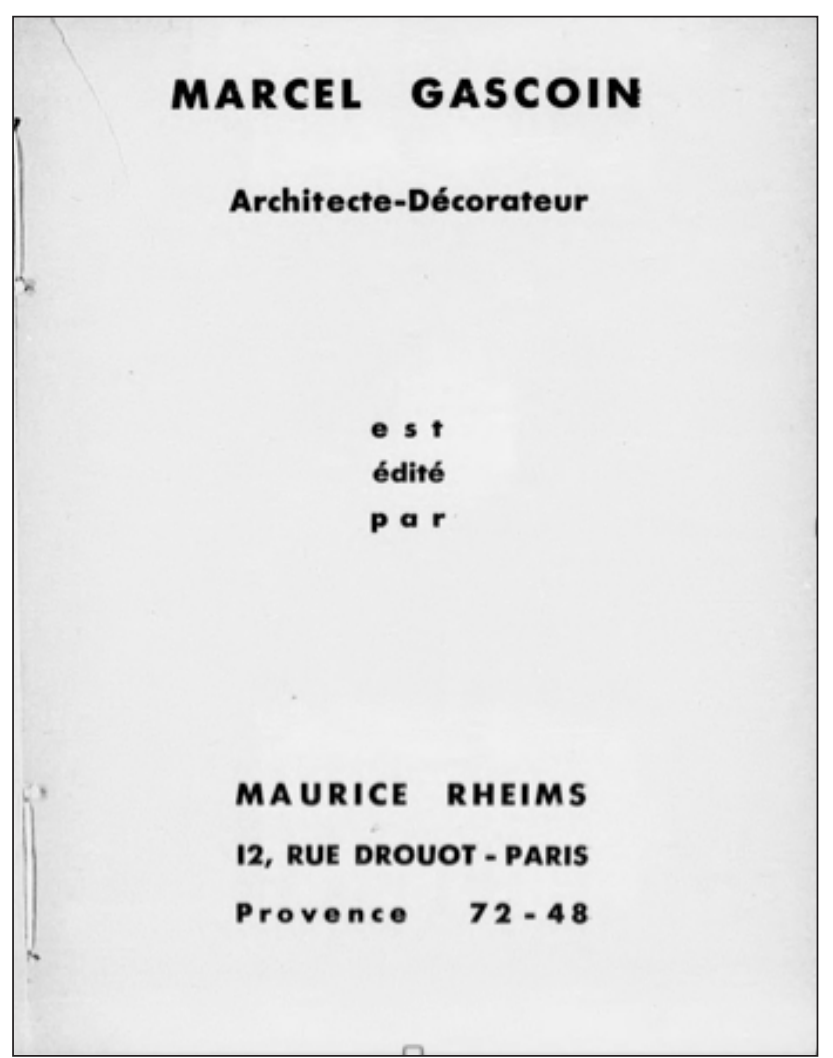

Figure 7 - Annuaire du catalogue de l'Exposition de l'Union des artistes modernes, Musée des Arts décoratifs, Pavillon de Marsan, juin-juillet 1930, Paris. Source: Bibliothèque Nationale de France. 
Pour se faire, il s'identifie à la figure prestigieuse de l'architecte formé à l'École nationale supérieure des beaux-arts. II s'inscrit dans la continuité d'une certaine pensée du décor total et rationaliste d'obédience viollet-le-ducienne. Déjà le théoricien des jardins André Véra écrivait en 1912 «(...) il s'agit en quelque sorte de faire naïvement œuvre d'architecte ». ${ }^{65}$ En 1913, Maurice Dufrêne rapproche le décorateur de l'architecte.

Le 'décorateur complet' est plus près de l'architecte que du peintre ou du sculpteur. (...) nous sommes des architectes d'intérieurs, nous imaginons, créons, mais comme les architectes nous ne pouvons édifier, comme eux il faut nous servir d'intermédiaires, d'exécutants industriels et ouvriers que nous dirigeons. (...) II y a une nécessité impérieuse pour les décorateurs de connaître à fond non seulement leur tâche de compositeur et leur mission d'artiste, mais les métiers qu'ils emprunteront, les techniques qu'ils traiteront afin de concevoir selon eux et par eux. ${ }^{66}$

En 1937, Francis Jourdain, membre de l'UAM, préfère le terme d'architecte d'intérieur qui organise l'espace. ${ }^{67}$ Quant à l'architecte-décorateur Louis Süe, il ne commence pas à concevoir en décorateur mais applique les arts majeurs "selon un point de vue décoratif". 68 Le jeune Marcel Gascoin, lui aussi, trouve "impropre" le terme décorateur qui ne traduit pas l'organisation de l'espace ni l'élargissement des programmes. Le souci du décorateur "n'est pas d'orner nos demeures, mais bien plutôt de les accorder avec la vie. (...) II sait (...) aussi bien transformer et rajeunir des 'bistrots' provinciaux, décorer un appartement bourgeois, que collaborer dans une entreprise industrielle ". ${ }^{69}$ Néanmoins, la figure idéalisée de l'artiste-artisan a survécu pendant la crise des années 1930 fortement marquées par le repli sur les valeurs artisanales. ${ }^{70}$ À cette figure sacralisant le savoir-faire national et le geste créateur, s'oppose presque diamétralement celle de "l'animateur moderne" que propose le décorateur Maurice Barret en 1935. II ne convient plus seulement de rapprocher l'ensemblier de la figure de l'architecte, maître d'oeuvre par excellence, mais de remettre en question sa suprématie.

Pour la première fois l'architecte-décorateur tente une collaboration totale avec l'industrie. Pour la première fois, il abandonne son pouvoir de 'créateur' au profit d'un nouveau rôle à jover : celui d' 'animateur' (...). Redisons donc une fois de plus que le monde des objets (cette notion s'étend à tout ce qui entre dans la maison) n'appartient plus à l'artiste-décorateur. Ce monde ne sera plus désormais que la résultante et la coopération de recherches collectives, où l'industriel, le technicien, l'organisateur, etc. auront partie liée avec le créateur-dessinateur de formes et d'objets (...) ».71

La re-définition des pratiques professionnelles dans tout le processus de la création des formes industrielles questionne toujours. La continuité entre les arts décoratifs et la création industrielle s'annonce impossible.
65. Vera (1912, p. 66).

66. Dufrêne (1913, p. 28).

67. Jourdain (1937).

68. Champigneulle (1934, p. 300).

69. Moutard-Uldry (1937, p. 147).

70. Iribe (1931).

71. Barret (1935, p. 26-27). 
72. Pour un panorama du mobilier de cette époque voir le catalogue de l'exposition Mobi Boom (Forest, 2010), éponyme du 22 septembre 2010 au 2 janvier 2011, Les Arts Décoratifs, Paris.

73. Parmi les membres fondateurs de l'Union, citons René Prou, Maurice Pré, Janette Laverrière, Jacques Hitier, Paul Beucher.

74. Ce projet de reconnaissance professionnelle nationale, porté par Maurice Dufrêne, est évoqué dans les textes de l'Assemblée générale de la SAD qui s'est tenue en Février 1942

75. La SDF est devenue en 1978 le syndicat patronal de l'Union nationale des architectes d'intérieur Designers (Unaid). La multiplicité des groupements donne une certaine illisibilité à la représentativité professionnelle.

76. Document de l'UACDE provenant d'archives privées relatives aux syndicats qui appartenaient à Jacqueline Lecoq. Elles ont été aimablement données à Béatrice Grondin. Pour faciliter la lecture des notes de bas de page, nous nommerons ces documents Archives JL-BG.

77. Archives SAD 1955 , Bibliothèque des arts décoratifs.
UNE PROFESSIONNALISATION QUI ENGAGE DE NOUVELLES TERMINOLOGIES

\section{Une mobilisation collective pour mieux définir les pratiques}

Au début des Trente Glorieuses, ${ }^{72}$ les revendications professionnelles se construisent autour du statut de "créateur" de modèles et d'architectures intérieures. Les décorateurs doivent identifier des compétences spécifiques pour tenter de parachever leur processus de professionnalisation auprès des pouvoirs publics. Des ensembliers renommés fondent en 1946 I'UADCE, ${ }^{73}$ après la création en 1939 du Syndicat Professionnel des artistes décorateurs (SPAD). Déjà en 1932, le viceprésident de la SAD, l'architecte Joseph Hiriart, engage des réflexions sur le statuł des ensembliers dans le contexte de crise économique et la perspective de I'Exposition internationale de 1937. Quelques années plus tard, le projet d'un Ordre des Artistes décorateurs ne voit pas le jour. ${ }^{74}$

Après guerre, les processus de reconnaissance officielle reprennent. Face aux urgences sociales, l'initiative de l'UADCE est significative de la volonté d'élargir les commandes dans le champ de l'habitation. Elle souhaite se doter d'une identité de groupe professionnel qualifié, et cela, dans une organisation plus fédératrice que la $S A D$, qui ne regroupe pas exclusivement des ensembliers, ou la Société des Décorateurs français (SDF) fondée en $1947^{75}$ qui accepte des décorateurs antiquaires. Cette union de créateurs "de toutes tendances esthétiques modernes" proclame en 1952 que le décorateur allie compétences techniques et artistiques (Figure 8 et 9). Par sa désignation et les pratiques de ses membres, cette association rassemble des créateurs de modèles, d'espace et d'ambiance qui souhaitent concevoir le "cadre de la vie contemporaine".76 Mais pour répondre aux besoins de la Reconstruction et l'augmentation du volume de la production de bâtiments, de nouvelles collaborations étroites devraient être redéfinies avec les architectes. Afin de garantir leur expertise spécifique, leur complémentarité professionnelle et leurs débouchés, les décorateurs souhaitent une protection de titre. Mais depuis l'amendement voté par le gouvernement de Vichy en décembre 1940, nul ne peut porter le titre d'architecte s'il n'est pas titulaire d'un diplôme et inscrit à l'Ordre. Cependant, la définition et la protection du titre pour exercer cette profession libérale n'octroient pas le monopole de son exercice. En 1955, le président de la SAD André Charles Jean Renou adresse une "Lettre aux architectes". Les décorateurs, écrit-il, sont « les spécialistes de l'intérieur qui voudraient bien s'appeler architectes d'intérieur et non plus décorateurs (...) Dans l'habitat moderne, la spécialisation est obligatoire ${ }^{7} .7$ 
Dans le catalogue du SAD en 1956,78 la fonction du "spécialiste de I'intérieur" consiste à installer et ordonner tout ce que l'industrie invente. ${ }^{79}$ Quelques années après Jean Royère, 80 le créateur Joseph-André Motte, ${ }^{81}$ interrogé par le critique Pascal Renous, revendique clairement une autre désignation professionnelle: "Je suis de ceux qui s'affirment architecte d'intérieur, créateur de modèles, et non pas 'décorateur' dans le sens de virtuose en décor divers, maquilleur, imagier, ornemaniste, que ce terme impropre suggère immédiatement».82 En 1959, André-Louis Pierre, vice-président de la SAD et secrétaire général adjoint de la CTI diffuse assez largement son article intitulé "De la qualification de l'artiste décorateur créateur". Les enjeux terminologiques animent aussi les débats du Congrès international d'esthétique industrielle de Stockholm. Si la définition d'esthétique industrielle et de design mobilise la scène internationale, certains acteurs en France, notamment à la SAD, cherchent à
78. Dans le catalogue du Salon de 1956, la SAD s'affirme comme une société qui rassemble tout le " domaine des Arts Appliqués à l'industrie ". La participation des industriels fut croissante comme en témoigne le lancement du concours de l'Aluminium Français en 1952-1953 ou encore les partenariats en 1959 avec les Établissements Agache, l'Aluminium Français, De la Rue Formica, ou encore les fabricants de glaces et verre Saint Gobain, Boussois et Aniche miroiterie de la S.A.G.A.I.S. Ils sont représentés aux côtés de la

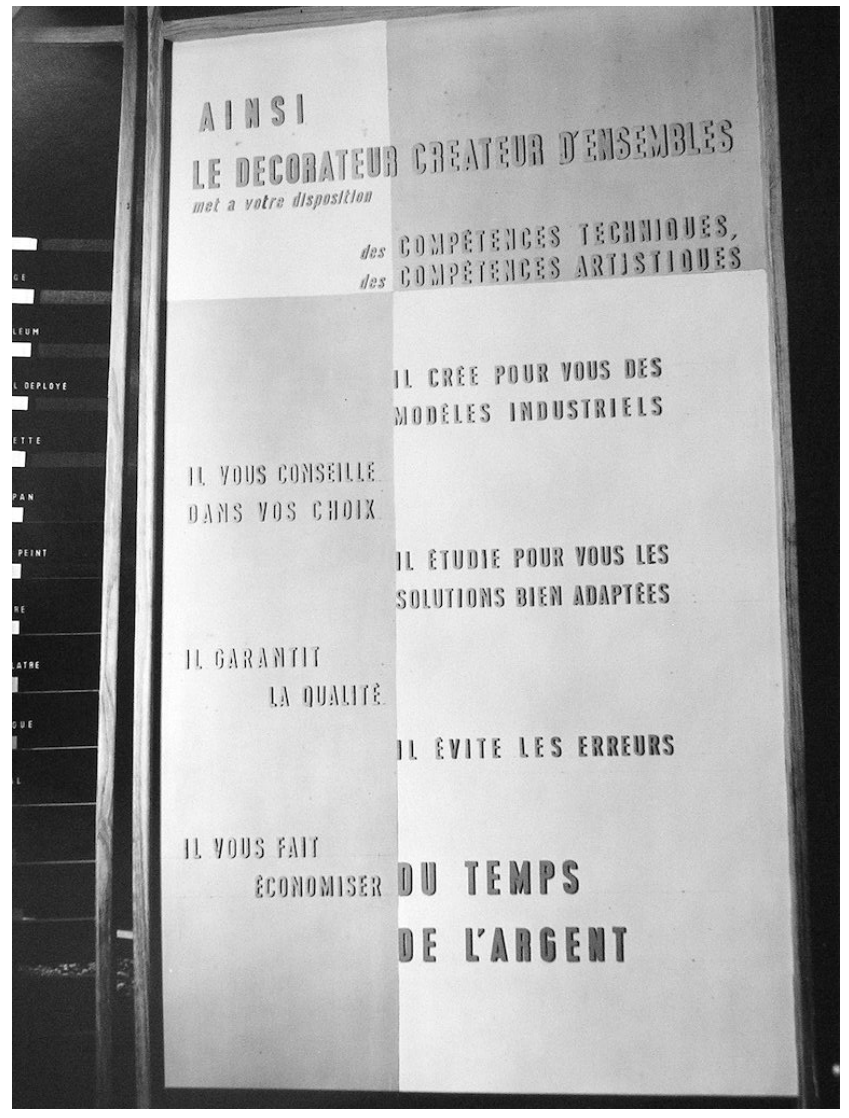

Figure 8 - Panneau de propagande de I'UADCE présenté au Salon des artistes décorateurs en 1952. Source : Inv. COL 9939-8-1-62, Musée des arts décoratifs, Don Annick Collas 1993, Paris. Photographie : Jean Collas.

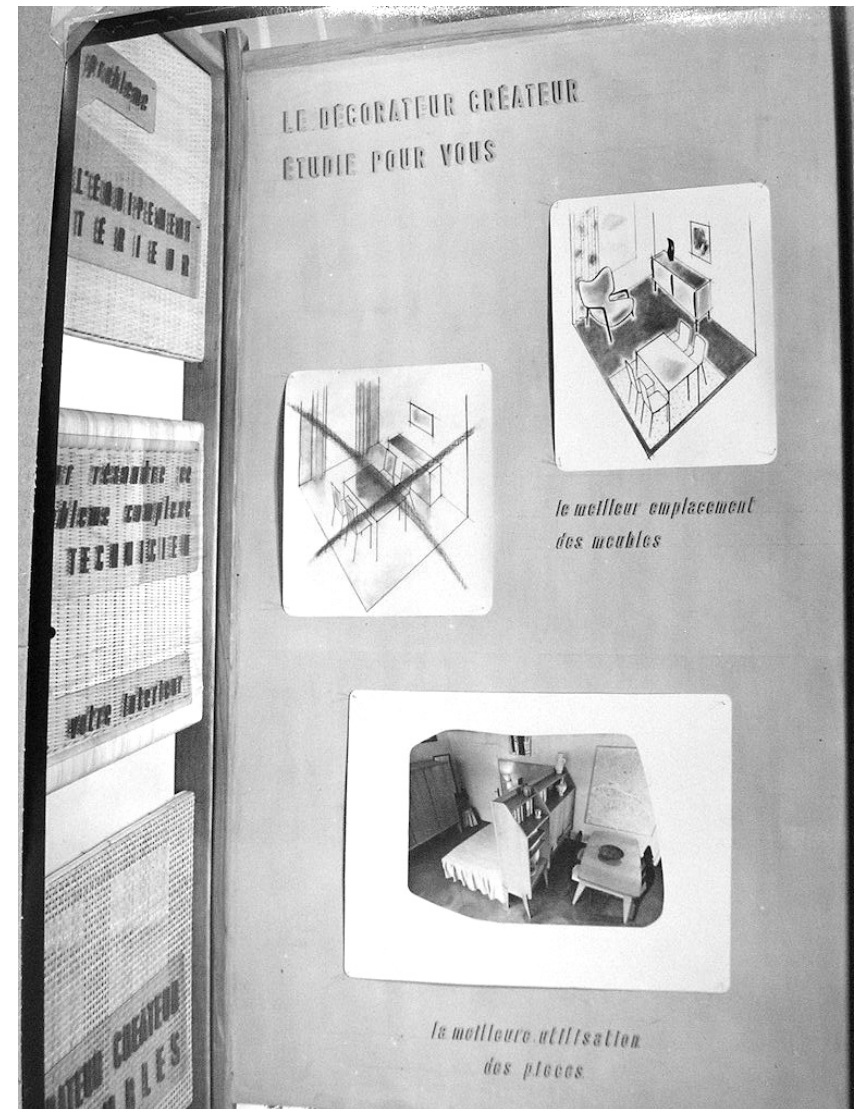

Figure 9 - Panneau de propagande de I'UADCE présenté au Salon des artistes décorateurs en 1952. Source : Inv. COL 9939-8-1-63, Musée des arts décoratifs, Don Annick Collas 1993, Paris. Photographie : Jean Collas. 
Fédération nationale des importateurs, négociants, industriels et commissaires en bois exotiques, coloniaux et américains, les Papiers Peints de France et les Établissements Delaroière.

79. Catalogue du 40e Salon des artistes décorateurs, p. 7.

80. Royere (1948).

81. Joseph-André Motte (1925-2013), cofondateur de l'ARP (Atelier de recherche plastique) avec Michel Mortier et Pierre Guariche de 1954 à 1957 , figure de proue de la nouvelle génération du meuble industriel, fut membre du Comité de la SDA en 1958, vice-président en 1961 et président de 1963 à 1965. Il fut responsable de l'architecture générale du Salon en 1959 avec R. J. Caillette, puis en 1961 avec J. Dumont, A. Philippon, E. Fermigier, A. Monpoix, J. Lecoq et A. Philippon.

82. Renous (1969. p. 26).

83. Etienne-Henri Martin (1905-1997), grande figure de la décoration française de l'entre-deux-guerres et de la période de transition de la Reconstruction, fut vice-président de la SAD de 1955 à 1958 puis président de 1958 à 1963, succédant au décorateur ensemblier André Renou.

84. Rapport moral du président Etienne-Henri Martin, Assemblée générale du 16 décembre 1959, p.5, archives SAD 1959. spécifier les compétences des artistes décorateurs dans une nouvelle économie

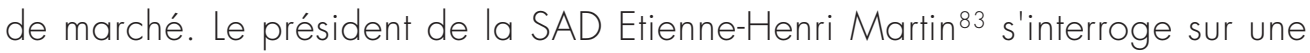
concurrence professionnelle qui résulterait de l'élargissement des pratiques dans la conception des modèles.

II est apparu que, dans la plupart des pays étrangers, les créateurs de modèles sont placés sous le signe de l'esthétique industrielle et, en définitive, exercent leur métier aussi bien pour étudier des machines, des objets usuels, que des éléments de l'équipement intérieur (meubles, appareils d'éclairage, tissus, couverts, céramique, etc.). On peut donc se demander si ce mouvement constitue un mouvement concurrent, et si l'organisation de nos professions, en France demande à être révisée. A moins, qu'au contraire, les Artistes Décorateurs soient suffisamment armés pour que tout ce qui concerne l'Habitat reste leur domaine. ${ }^{84}$

L'inquiétude d'Etienne-Henri Martin se comprend d'autant mieux que les présentations des Salons du SAD ne manifestent pas de changement radical survenu dans la production française (Figure 10).

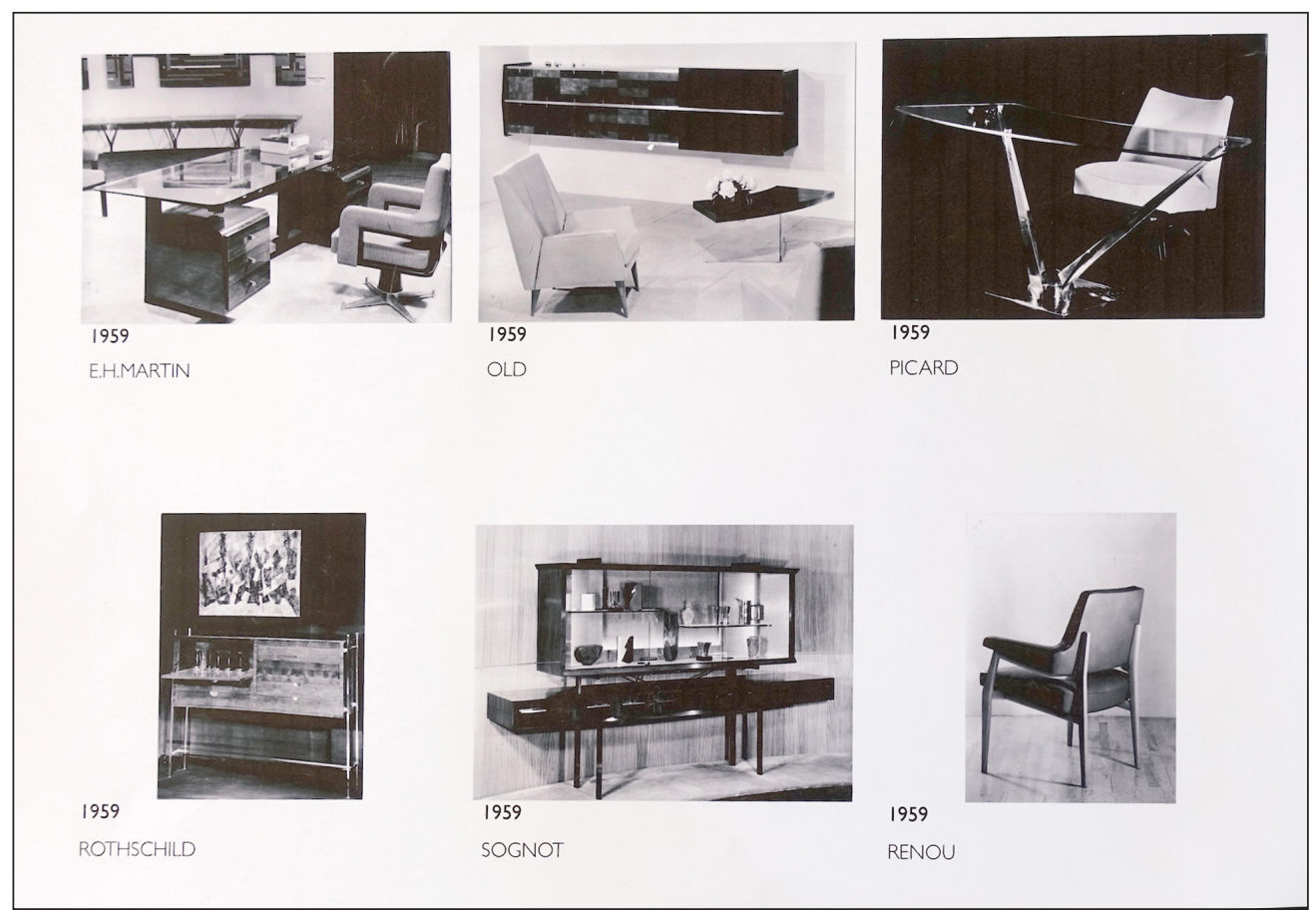

Figure 10 - Planche issue de la maquette d'un projet de publication retraçant l'histoire des Salons de la SAD au cours de la seconde moitié du XXe siècle. Source: Archives Société des artistes décorateurs, Bibliothèque des arts décoratifs, Paris 


\section{Des figures façonnées par un nouveau contexte socio-économique et industriel}

Dans une enquête de 1960 organisée par I'UADCE, ${ }^{85}$ le décorateur Louis Baillon, membre actif de la SAD (Figure 11), décrit l'évolution de la profession des décorateurs modernes comme l'aboutissement des idées progressistes de René Gabrie| 86 et de Francis Jourdain :

(...) on voit l'objet luxueux aujourd'hui s'aligner sur l'objet de série. Nos grands aînés, qui se voulaient Artistes Décorateurs avec majuscules, travaillaient pour la grande bourgeoisie, installant, meublant, décorant de vastes et somptueuses demeures avec luxe, raffinement, prestige. Depuis, aucune structure sociale n'étant fixe, les revenus se sont déplacés, étalés, les grandes fortunes raréfiées, pendant que de nouvelles couches de la société aspiraient au mieux être et au confort. En parallèle, pour répondre à ce mouvement, l'artisanat du meuble et du siège se transformait peu à peu en industrie, s'équipant d'une machinerie de plus en plus perfectionnée pendant qu'apparaissaient de nouveaux matériaux et de nouvelles techniques. Ce mouvement social et technique détermina une véritable révolution dans notre profession et la divisa en deux grands courants extrêmes $(. . .)^{87}$

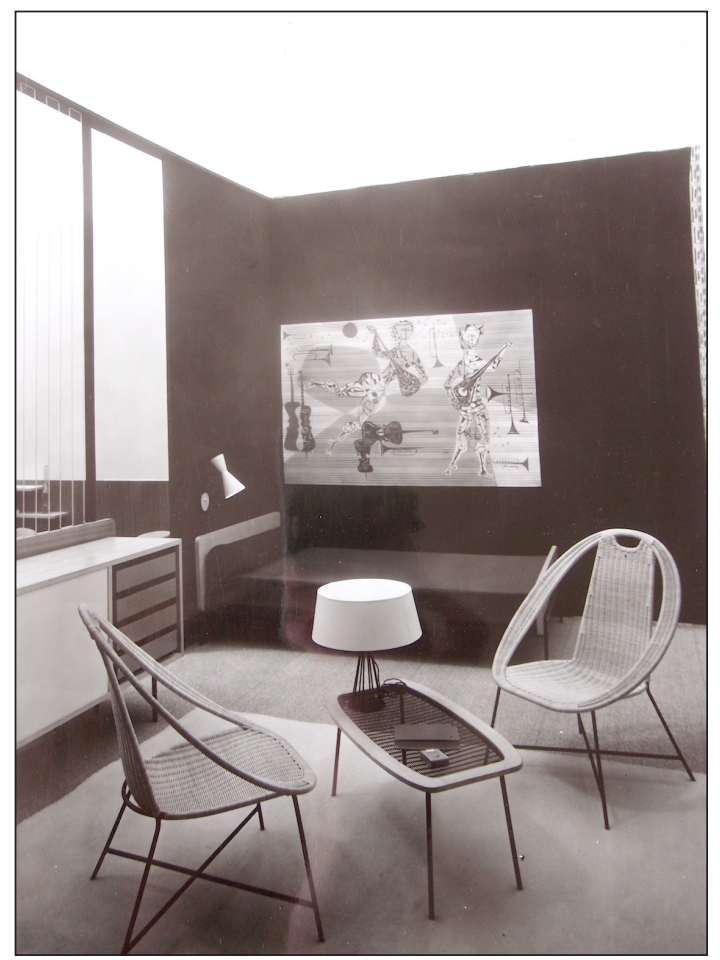

Figure 11 - Louis-Auguste Baillon, chambre d'étudiant, collaboration de Caillat, édition Ełs Goubrin, présentée au 38e Salon des artistes décorateurs, organisé sous le patronage du Ministre de l'éducation nationale et intitulé "La jeunesse et le cadre de la vie", 25 mai-4 juillet 1954, Grand Palais des Champs-Elysées, Paris. Source: Archives Société des artistes décorateurs, Bibliothèque des arts décoratifs, Paris
85. Archives JL-BG.

86. René Gabriel (18901950) fut président de la SAD de 1944 à 1947. Comme d'autres figures majeures de l'entre-deuxguerres orientées vers la série, il exerça une influence capitale sur une génération de créateurs par son enseignement à l'Ecole nationale supérieure des arts décoratifs (Gencey, 2016).

87. Archives JL-BG. 
88. Citons par exemple Maxime Old, Jacques Adnet, André Arbus, Jacques Quinet. Cf. Amic (1983); Bony (1983); Favardin (2012).

89. Archives JL-BG
Louis Baillon distingue les rares ensembliers, qui poursuivent la tradition du luxe de l'entre-deux guerres, ${ }^{88}$ des jeunes décorateurs inspirés des pays d'Europe du Nord suivant de près les développements de l'architecture moderne. Ouverte aux besoins réels de la collectivité et aux collaborations industrielles, cette génération expérimente des champs d'application comme le tourisme (hôtellerie), le commerce (installations de magasins), l'administration d'Éłat ou privée (installations de bureaux) (Figure 12).

Aussi ayant à considérer l'activité humaine non plus seulement à l'échelle de l'individu, mais à celle du groupe ou même de la société ayant à résoudre, non plus des problèmes de prestige mais d'organisation de collectivités, notre profession doit-elle être ouverte à tous les problèmes humains de notre temps, tout en se rapprochant de l'architecture jusqu'à en constituer la suite nécessaire, de façon à ce que, de l'urbanisme à l'objet usuel, l'enchaînement soit logique et harmonieux. ${ }^{89}$ (Figure 13)

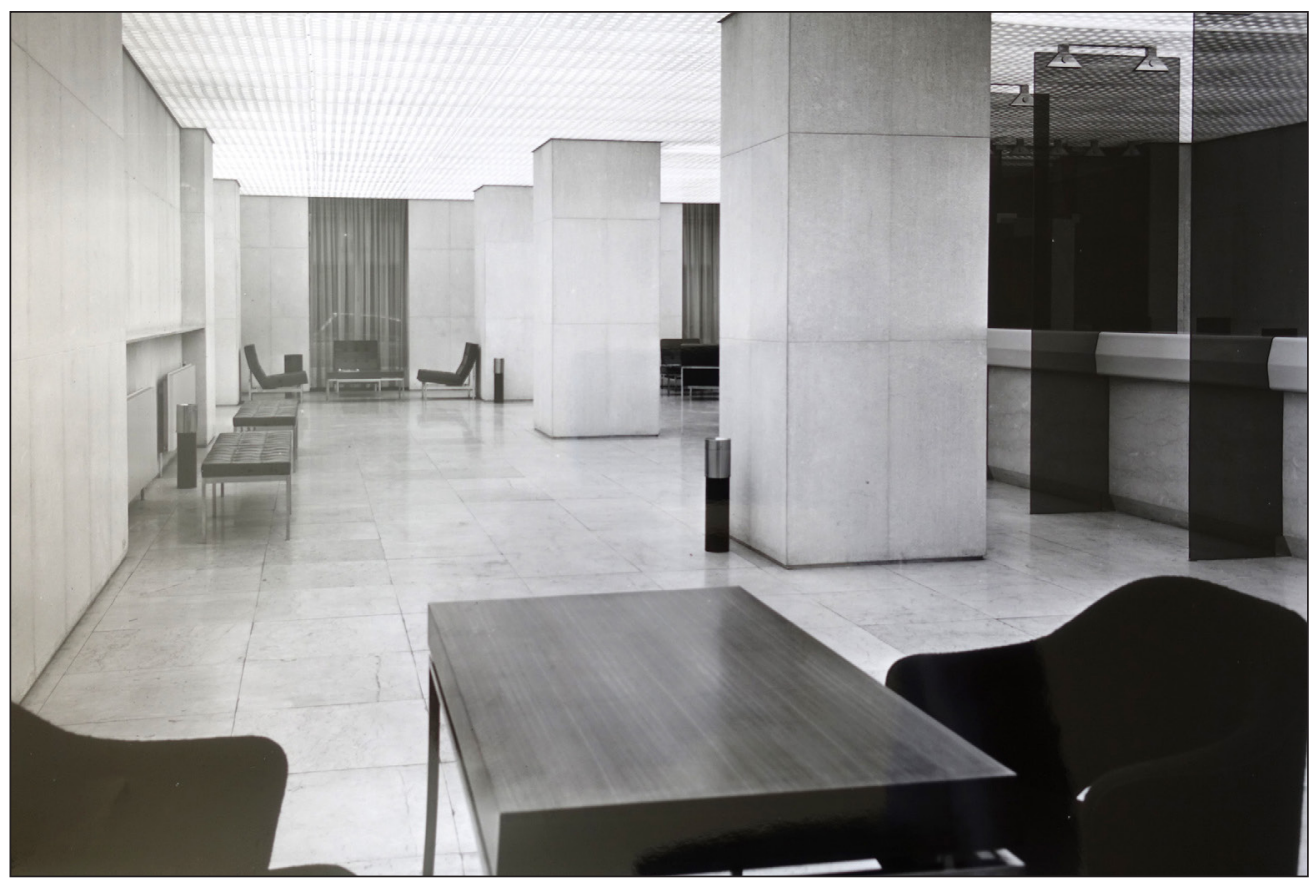

Figure 12 - Jacques Dumont (collaborateur Philippe Leloup), hall de la direction d'une société de crédit, 1966. Source: Archives Société des artistes décorateurs, Bibliothèque des arts décoratifs, Paris 


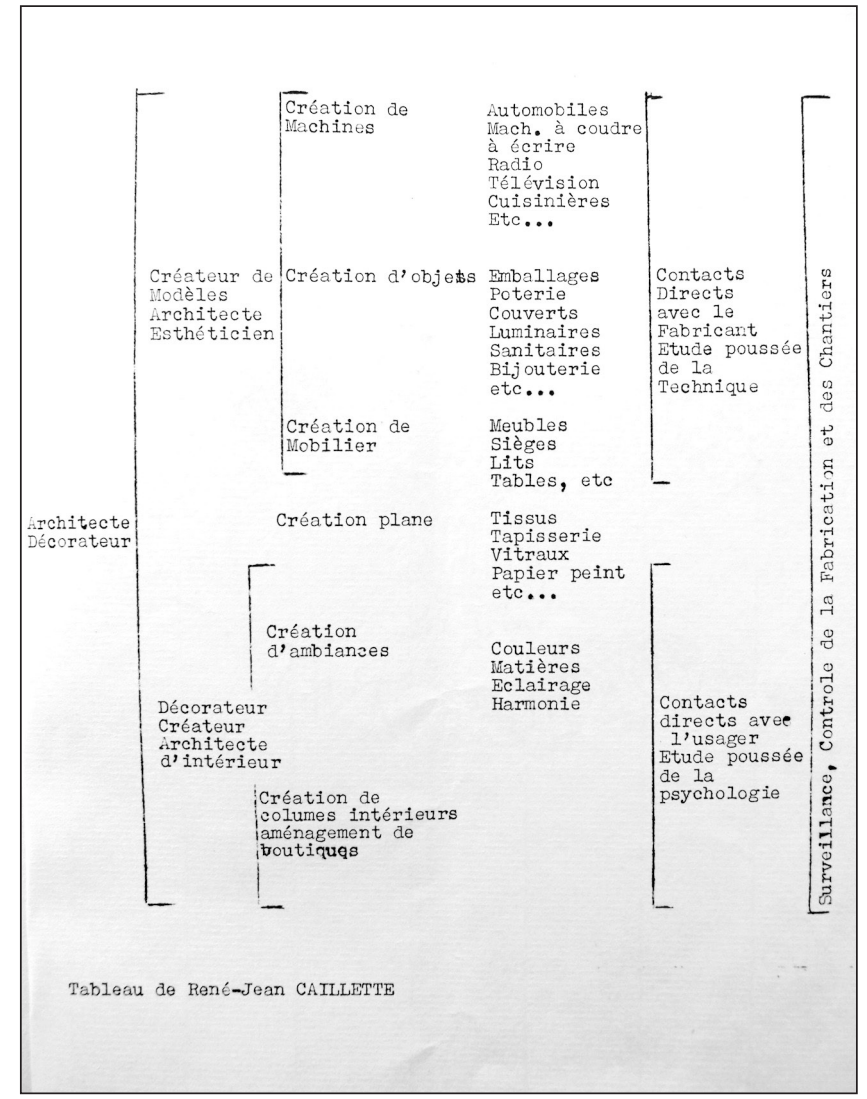

Figure 13 - Tableau de René-Jean Caillette extrait du rapport de la Commission de définition de la profession de I'UADCE, 1960. Source: Archives personnelles Béatrice Grondin

Jusqu'au milieu des années 1960, le goût pour l'éclectisme et le pastiche des élites et des classes populaires, ${ }^{90}$ la frilosité des industriels du meuble à l'égard de l'"esthétique contemporaine", 91 la concurrence étrangère toujours aussi vive, la commande publique prestigieuse plus restreinte tout comme celle de la clientèle privée, contraignent fortement les initiatives des créateurs modernes. Par ailleurs, la figure du décorateur "tailleur sur mesure" possédant sa boutique tend à disparaitre. Selon Etienne-Henri Martin, "Nous allons devenir, en mettant les choses au mieux, des espèces de chefs d'orchestre, des coordinateurs. II nous appartiendra d'organiser un intérieur avec des éléments prélevés à droite et à gauche ". ${ }^{92}$ Pour le décorateur Jacques Dumont, ${ }^{93}$ l'ensemblier ne disparaît pas mais s'affirme plus technicien:

L'esthétique industrielle a modifié le rôle du décorateur, qui, outre son activité de créateur de modèles, devient maintenant, un ensemblier, titre qu'il portait déjà, mais dans une acceptation très différente. (...) C'est précisément au décorateur que revient de choisir et d'assembler les objets qui ont entre eux une certaine résonance pour les intégrer convenablement dans le milieu architectural dont il est l'auteur et le technicien. ${ }^{94}$
90. Cf. Baudrillard (1968).

91. L'expression est extraite de la documentation générale de l'Association des Créateurs de Modèles en Série qui l'emploie fréquemment. (Archives Nationales ACMS/CAIM 19930521/86-88)

92. Renous (1969, p.44).

93. Jacques Dumont (19252009) a collaboré avec l'architecte Pierre Patout et fut l'un des fondateurs de l'Institut d'Esthétique Industrielle avec Jacques Viénot. Il a été membre du comité directeur de l'UAM, fondateur avec André Hermant de "Formes Utiles " en 1949. Il s'est penché sur le mobilier en série dès 1947 et mène des recherches assez proches de René Gabriel et Marcel Gascoin. Exposant depuis 1944 à la SAD, il fut commissaire du Salon en 1953, vice-président de la Société de 1954 à 1961 et président en 1969. Il a été choisi pour représenter le meuble français de luxe à la Triennale de Milan en 1953.

94. Dumont (1963, p. 67). 
95. Archives Nationales ACMS/CAIM 19930521/8688

96. Précisons que dans la documentation de l'association, le terme décorateur es t paradoxalement employé tout autant que créateur de modèle. Il en va de même pour la documentation de l'UADCE.

97. Le Ministère de la Reconstruction et du Logement organise en 1953 avec le Centre Technique du Bois (CTB) le premier concours du meuble de série. Puis le CTB organise un deuxième concours en 1955.

98. Dans les statuts de l'Association, l'intérêt sur le plan moral de la signature se résume ainsi : "Rendre à l'artiste sa véritable place dans la profession ; revaloriser son talent, devenir une source d'émulation, donner à de nouveaux talents des possibilités plus grandes de se révéler, susciter l'intérêt du grand public qui pourra comparer et mieux réaliser la différence entre les œuvres signées et celles qui ne le sont pas. "(Archives Nationales ACMS/CAIM 19930521/86-88)

99. Les "décorateurs" sont mal identifiés selon la Convention collective nationale de l'ameublement de 1956, dont les termes ne changent guère en 1971.

100. Le vocable "décorateur" n'est toutefois pas récusé dans le débat sur la formation. Par exemple, Le 16 janvier 1959, les membres de l'UADCE se sont réunis au Pavillon de Marsan afin de débattre sur le défaut d'un titre officiellement reconnu et protégé par la loi. La constitution d'un Comité supérieur consultatif des arts appliqués est envisagée

\section{La fin du décorateur?}

Les termes "artiste", "ensemblier" et "décorateur" disparaissent de la désignation syndicale en 1961 des CAIM. Ce groupement est né de la fusion de I'UADCE et de l'Association des créateurs de modèles en série (ACMS). Cette dernière, fondée en $1952^{95}$ sous la présidence de Marcel Gascoin, regroupe des créateurs de modèles 96 qui tentent de promouvoir un ameublement rationnel de qualité et en série, ${ }^{97}$ dans un cadre juridique favorable à la reconnaissance du créateur. Elle souhaite normaliser les rapports avec les fabricants et négociants par la mise au point d'un contrat d'édition type. Elle allie politique de marque (ACMS) et droit à la signature ${ }^{98}$ en réaffirmant le statut du créateur. Les buts de l'association témoignent aussi de la place que ces artistes ${ }^{99}$ veulent prendre dans "l'amélioration du cadre de vie". Mais les rares débouchés dans ce domaine conduisent les créateurs de modèles à s'orienter davantage vers l'architecture intérieure. La plupart d'entre eux réalisent leur prototype en auto-édition. L'ACMS est alors dissoute en 1961 pour fusionner avec I'UADCE donnant naissance au CAIM.

En 1961, les membres du CAIM font disparaître le mot décorateur jugé impropre par les décorateurs eux-mêmes mais aussi par les pouvoirs publics. Insatisfaisant pour traduire les nouvelles dynamiques de pratiques et les nouveaux enjeux de société, il renvoie toujours à des professions diverses ou des pratiques amateurs. Ainsi, pour exprimer la reconnaissance d'expertise souhaitée, la désignation "architecture" (et non architecte) est adoptée suite à l'enquête menée en 1960 par I'UADCE, 100 avant sa dissolution et la fondation du CAIM. Plus de 90 membres ont répondu à la Commission "Définition de la profession" regroupant Louis Baillon, Antoine Philippon, "101 RenéJean Caillette 102 et Jacques Altayrac. ${ }^{103}$ La plupart des membres contestent l'usage "artiste décorateur" qui ne désigne pas clairement les pratiques de l'aménagement intérieur. Ce titre général n'englobe pas l'activité de créateur de modèles qui, pour les intéressés, ne relève plus de la profession du décorateur. La difficulté consiste à trouver une terminologie rassemblant les diverses branches de la conception de l'habitat. Si "artiste décorateur" et "décorateur" apparaissent galvaudés, les termes "créateur d'ensemble" ou "ensemblier" ne couvrent pas la dimension architecturale et spatiale de l'activité. Contrairement à d'autres pays qui utilisent les désignations architecte d'intérieur et designers, les créateurs français cherchent une équivalence. A la question posée «Estimez-vous que l'exercice de la profession de 'décorateur créateur de modèles d'équipement mobilier et d'ensemble' présente aujourd'hui un caractère particulier, qui différencie cette profession des autres groupées plus généralement sous le nom d"artistes décorateurs' et sur ce point, apparente cette profession à celle d'un architecte spécialisé? », les personnalités ont répondu unanimement par l'affirmative. Pour Bernard De Swarte «La profession est en effet tout à fait particulière. 
Je suis d'accord sur la comparaison avec celle d'architecte spécialisé.» Roger Landault104 l'affirme " oui, nous sommes plus architectes et esthéticiens que 'décorateurs' ». Claude Bailly précise «le nom reste à trouver...l'important est que la profession soit parallèle à celle d'architecte et reconnue comme telle. » Pour Charles Ramos,

Il n'y a ni 'artiste' ni 'décorateur'. Nous sommes des architectes spécialisés, nous sommes des artistes dans la mesure où est artiste un architecte. (...) L'UADCE paraît prendre conscience de tout cela en pensant à une organisation de notre profession et promouvoir non plus des décorateurs qui n'ont plus rien à 'décorer' mais des 'artistes techniciens'.

Pour Serge Lesage "ce n'est plus une profession d'artiste mais de spécialiste.»105 Enfin, Jacques Mottheau, membre fondateur de I'UADCE et du CAIM, contributeur de l'Encyclopédie Pratique de la Construction et du bâtiment de 1958, conclut:

Notre profession présente aujourd'hui un caractère particulier, qui la différencie nettement de celle d' 'artiste décorateur' en général, étant bien entendu, cependant, qu'il subsistera toujours un commun dénominateur propre à toutes les disciplines artistiques: La Création. ${ }^{100}$

Suite à cette enquête, la désignation du CAIM est choisie. ${ }^{107}$ En 1964, une définition professionnelle s'impose dans les textes syndicaux. Malgré l'absence de reconnaissance officielle du titre, les architectes d'intérieur s'identifient ainsi:

(ils) partent de la structure d'immeubles ou fraction d'immeubles, organisent et aménagent le cadre architectural de la vie privée ou collective des hommes, en harmonisent les volumes, les formes, les couleurs, les matériaux, et, dans cette perspective, créent, c'est à dire conçoivent de façon originale, étudient, mettent au point et exécutent ou font exécuter par l'industrie ou l'artisanat, tous équipements mobiliers et objets utilitaires ou d'agrément, d'emploi journalier ou de caractère exceptionnel.»108

Il semble qu'après la clarification des relations avec les industriels, ce soit celles avec les architectes qui deviennent plus urgentes à préciser. ${ }^{109}$ Pour y parvenir, le CAIM prend pour modèles des dialogues fructueux entre architectes et décorateurs. L'objectif est de définir idéalement leurs relations en vue d'un protocole 110 d'accord avec les syndicats d'architectes. ${ }^{111}$ Les opportunités de marchés publics, pendant les années 1960, offrent quelques collaborations. Le parcours de Jacques Dumont en est un exemple (Figure 14 et 15). L'architecte André Wogensky, dans une lettre du 16 avril 1968 adressée à la Direction de l'architecture, reconnaît l'importance de l'implication pour connaître toutes les questions relatives à l'enseignement des arts appliqués et pour préparer un projet de diplôme national unique de décorateur-créateur de modèles et d'ensembles.

101. Antoine Philippon (1930-1995) a présidé la SAD de 1970 à 1972 et exposé comme de nombreux membres de la SAD tout autant aux Arts Ménagers qu'à la SAD, avec Jacqueline Lecoq (1932). Il a participé à l'architecture générale du Salon avec Jeanne Couturier, André Monpoix et Pierre Paulin. Archives du Centre de documentation des Arts Décoratifs, Fonds Philippon Lecoq XXe, PL.

102. René-Jean Caillette (1919-2004), membre du groupe Saint-Honoré et du Groupe 4, cofondateur de l'ACMS, fut un défenseur du meuble de série. Il participa à l'architecture générale du salon en 1956 et fut membre du comité entre 1958-1959, responsable de la section "L'appartement du matin " au salon de 1961 et vice-président de la Société en 1965.

103. Le rapport est présenté lors du Conseil d'Administration du 6 mai 1960.

104. Après guerre, Roger Landault (1919-1983) a élaboré avec l'éditeur A.B.C du mobilier de série.

105. Toutes les citations sont extraites du rapport de l'enquête provenant des archives JL-BG.

106. Archives JL-BG.

107. Jacques Hitier, dans le bulletin CAIM de 1962 , l'atteste : " Il restait à trouver le vocable ; après bien des hésitations, ce fut enfin celui de créateurs d'architectures intérieures 
et de modèles qui répondait le mieux à nos aspirations. Ce titre résulte également d'une enquête qui fut ouverte auprès de nos camarades qui ont tous été unanimes à reconnaître qu'il s'agissait pour nous d'aménager des volumes intérieurs, de créer des modèles pour la vie de l'homme dans son habitation privée et collective, comme nous le rappelons dans la définition de la profession. Les vues et les buts à atteindre de notre jeune syndicat, restent ceux que nous nous sommes toujours fixés reconnaissance officielle d'un titre, création d'un diplôme unique, auxquels bien sûr nous porterons tous nos efforts (...) " Archives JL-BG.

108. Bulletin... (1964).

109. "Combien avons-nous souhaité que notre métier puisse s'exercer de concert avec ceux qui ont pour mission de créer et de bâtir. Combien avons-nous regretté souvent que l'architecte, absorbé par les responsabilités de son travail, ait omis d'appeler le spécialiste de l'installation intérieure qui aurait, par sa compétence, prolongé harmonieusement la réussite de l'ouvrage. Désemparés devant l'appellation de décorateur désignant une multitude de métiers indéfinis, l'architecte, nous connaissant mal et redoutant la mutilation de son œuvre, va ignorer ou quelque fois proscrire toute collaboration pour ce qui est de la décoration intérieure de l'ouvrage qu'il construit. Mais voici que beaucoup d'entre nous, à force de persévérance, ont établi avec de nombreuses agences d'architecture d'excellents rapports. De ces fructueuses collaborations, des œuvres importantes et variées ont du décorateur en amont du projet afin qu'il n'œuvre pas "contre l'architecture". L'architecture est indivisible et le décorateur, comme collaborateur, doit être placé sous l'autorité de l'architecte qui, lui aussi, se revendique créateur dans la maîtrise d'œuvre. Le syndicat du CAIM fait pression auprès des diverses Directions pour parvenir à de nouvelles règlementations (droits, barème de rémunération). Mais un courrier de Bernard Anthonioz de la Direction de la Création artistique adressé à la Direction de l'architecture, datant du 28 Août 1967, atteste de la subordination professionnelle:

En admettant que la spécialisation des différentes missions de l'architecte justifie la définition d'une nouvelle profession de créateurs d'architecture intérieure, il est certain que cette règlementation ne pourrait se faire qu'en fonction de celle déjà en vigueur pour les architectes. Le Service de la Création artistique a surtout à connaître les décorateurs en tant que créateurs de modèles. Les décorateurs se rapprochent beaucoup plus sur ce point des autres artistes des arts plastiques et leur profession ne semble pas appeler de règlementation particulière. »112

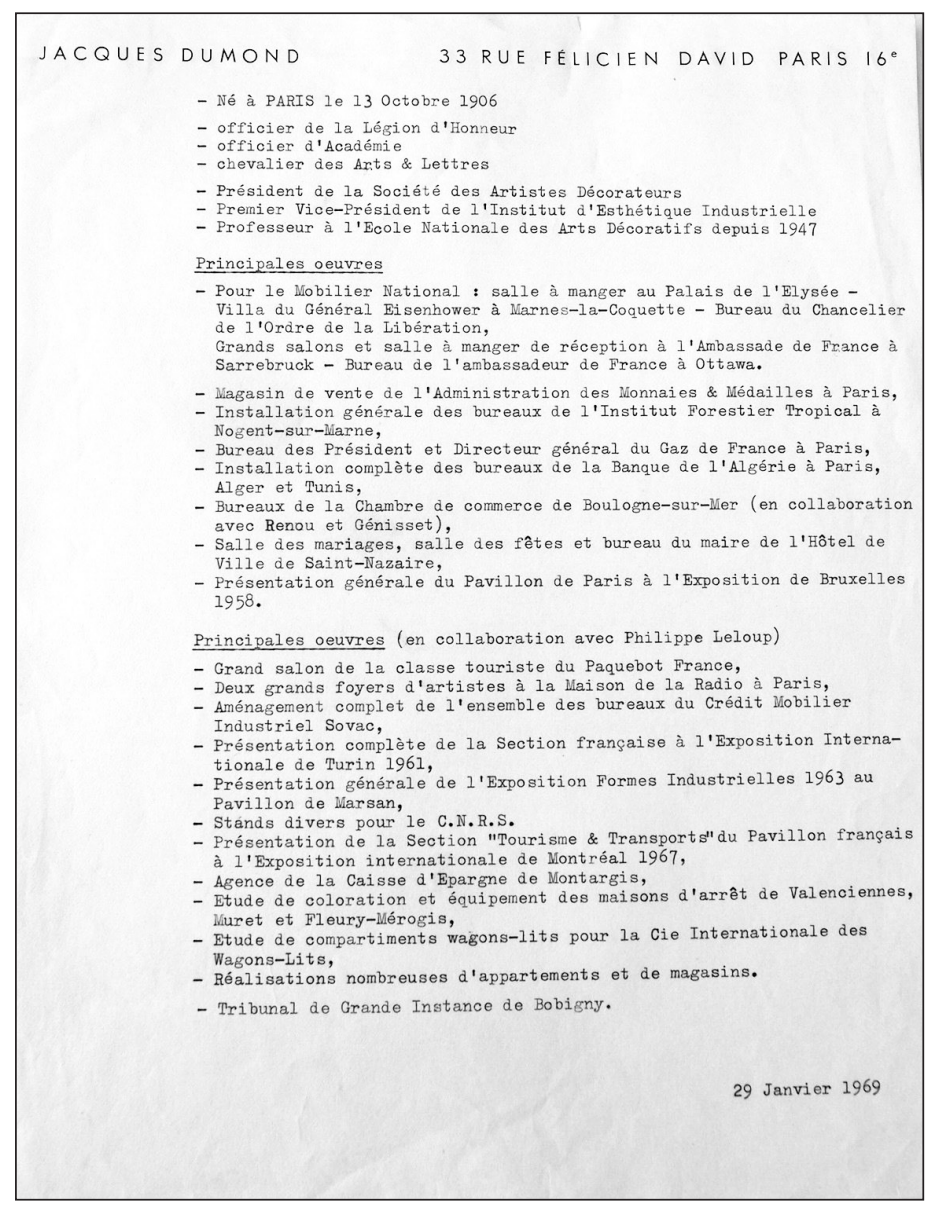

Figure 14 - Curriculum vitae de Jacques Dumont circa 1969. Source: Archives Société des artistes décorateurs, Bibliothèque des arts décoratifs, Paris. 


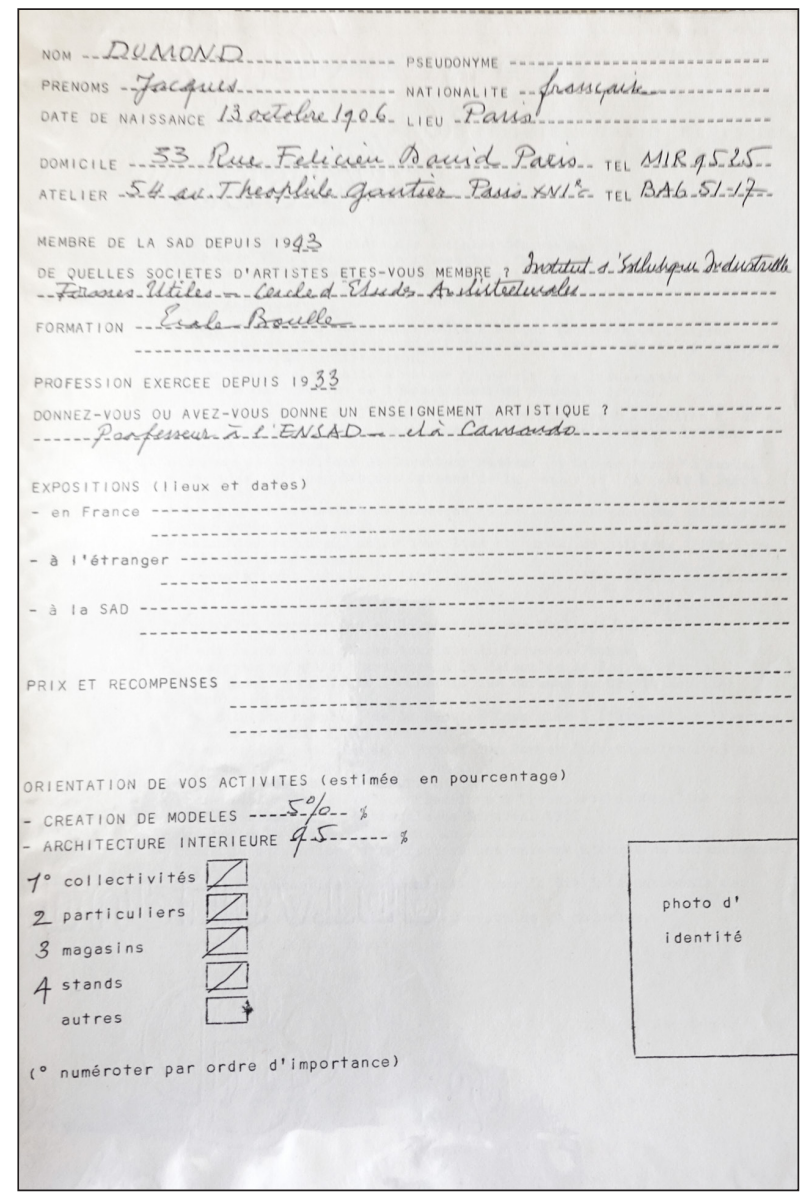

Figure 15 - Fiche de renseignement des sociétaires SAD, Jacques Dumont, circa 1969. Source: Archives Société des artistes décorateurs, Bibliothèque des arts décoratifs, Paris.

La Direction de l'architecture devra trancher sur le sort règlementaire des architectes d'intérieur. Or, la nouvelle loi sur l'architecture de 1977 ne permettra pas de faire consensus, d'autant plus que le recours à un professionnel pour l'aménagement intérieur n'est pas obligatoire. Malgré les obstacles que la définition en creux induit, l'architecte d'intérieur ${ }^{113}$ affirme encore en 1977 son expertise pluridisciplinaire dans le second œuvre.

L'architecte d'intérieur n'est pas que décorateur, avant tout ornementaliste, I'architecte d'intérieur n'est pas architecte mais doit néanmoins avoir une très bonne connaissance de I'architecture (...) ; l'architecte d'intérieur n'est pas un designer hautement qualifié mais doit être néanmoins apte à créer un certain nombre de modèles notamment dans le domaine du mobilier ; l'architecture intérieur n'est pas un graphiste mais doit néanmoins savoir utiliser les compétences de celvi-ci en le cadrant dans le domaine architectural. »"144 (Figure 16) abouti, prouvant ainsi l'opportunité de la collaboration Architecte et Décorateur-créateur. " (Bulletin...1964).

110. Courrier de Jacques Hitier, Président du CAIM, adressé aux membres du CAIM au nom de la Commission "Rapports Architectes-Décorateur Créateur", du 31 mars 1964 (Archives JL-BG)

111. La Commission "Reconnaissance de la profession-rapports avec les architectes est composée pour l'année 1964 de Jacques Dumond, Jacques Hitier; Jean Lesage, J. Maheu, Joseph-André Motte, Maurice Pré, Charles Ramos, André Preston e Francis Ullmann. Les relations entre le CAIM et les syndicats d'architectes restent à éclaircir, bien que l'histoire de la Société des Architectes Diplômés du Gouvernement (SADG) ait déjà fait l'objet d'une synthèse. (Dumont, 1989).

112. Archives Nationales ACMS/CAIM 19930521/86

113. Cf. Ollivier (2010).

114. Bulletin... (1977, p. 3). 
115. Société des Artistes Décorateurs (1961).

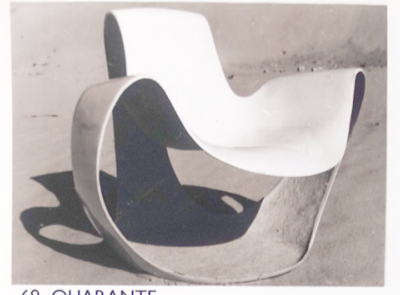

69 QUARANTE

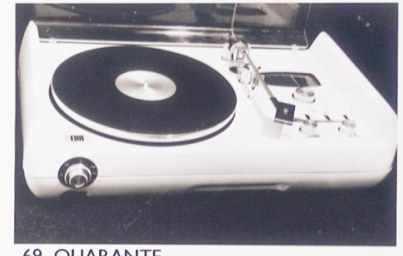

69 QUARANTE

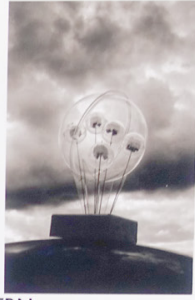

69 HALPERN

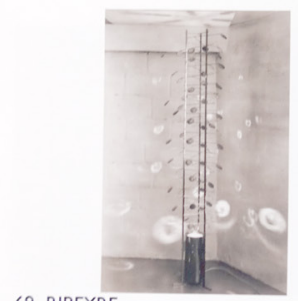

69 RIBEYRE

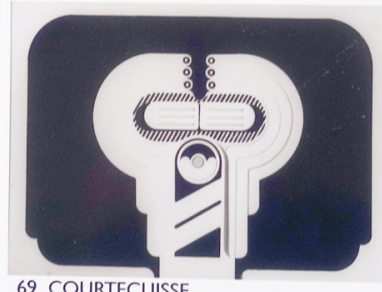

69 COURTECUISSE
69 ZUBLENA

Figure 16 - Planche issue de la maquette d'un projet de publication retraçant l'histoire des Salons de la Société des artistes décorateurs au cours de la seconde moitié du XXe siècle. Source : Archives Société des artistes décorateurs, Bibliothèque des arts décoratifs, Paris.

Le processus d'identification de l'architecte d'intérieur implique la reconnaissance de spécificités communes en terme de pratiques, de conditions d'exercice (en faveur du statut libéral par exemple) mais aussi de formation au sein des écoles d'art et de techniques. La SAD s'engage dans l'enseignement avec son président André Renou en 1955 puis Etienne-Henri Martin en 1961, en siégeant par exemple au conseil de perfectionnement de l'École des arts appliqués à l'industrie de Paris (lycée technique municipal), rue Dupetit-Thouars. Le procès-verbal de la séance du 23 janvier 196 1115 révèle les réformes de cette école après la direction de Jean Fressinet, en termes de recrutement des élèves et de contenus pédagogiques. En 1960, une nouvelle formation de dessinateur polyvalent et créateur de modèles est prévue sur un cycle de quatre années (mais seule la 2e année est mise en place). La formation d'un spécialiste, de cycle supérieur plus technologique, est envisagée. La 3e année fonctionnerait "comme un bureau d'étude", avec des professeurs de spécialités et des exercices de création étudiés dans les mêmes conditions que la profession. Prévue pour la rentrée de 1963, une 4e année de second cycle devait être plus ciblée sur les problèmes d'ensemble et d'espace "sous la forme de section de techniciens (qui) formerait en petit nombre des spécialistes à un niveau supérieur, élite de créateurs et de techniciens nécessaires à l'industrie et à l'artisanat dans toutes 
les spécialités (...)". ${ }^{116}$ Les élèves du nouveau Cours supérieur d'esthétique industrielle mis en place par Jacques Viénot dès 1956, suivent également le cours de Jean Prouvé au Centre national des arts et métiers (CNAM). Lors de ce Conseil en 1961, l'ingénieur M. Fayeton remarque que l'enseignement dans les écoles françaises n'est pas assez technique pour favoriser les collaborations avec les architectes, comparées aux écoles spécialisées suisses et allemandes. Si la question de la formation d'un technicien en architecture est bien en discussion, aboutissant au premier Brevet de Technicien Supérieur (BTS) en arts appliqués en 1962, l'approche polyvalente, technique et professionnalisante semble lacunaire. Toutefois, cette même année, Roger Tallon assure un enseignement d'esthétique industrielle à l' qui déjà, dans les années Trente, faisait appel à des architectes pour ses enseignements et délivrait un diplôme d'ensemblier-décorateur. Elle fut reconnue apte à enseigner l'architecture jusqu'en 1940, comme l'École Nationale Supérieure des Beaux-Arts et l"École spéciale d'architecture. Mais rappelons que l'École nationale supérieure de création industrielle (ENSCI) ne sera créée qu'en 1982. La formation se poursuit donc dans les bureaux d'études des "anciens" et en prise directe avec les industriels. De leurs côtés, les architectes diplômés par l'État à partir de 1968 ne dépendent plus de la section architecture de l'École nationale supérieure des beaux-arts mais des 12 "unités pédagogiques d'architecture" (UPA), de l'École Spéciale d'architecture ou encore des écoles de dessin régionales. L'enseignement de l'architecture connaît une remise en question profonde. Dans la pratique professionnelle, la séparation des compétences de programmation et de conception s'accentue tout comme la concurrence de nouveaux métiers au sein de la maîtrise d'œuvre. Cette situation plus complexe ne facilite pas la reconnaissance de ceux dont la pratique, comme l'écrit Jacques Vienot, " ne relève ni des beaux-arts, ni des arts décoratifs ni de la technique ». 117 D'ailleurs, c'est dans le nouveau contexte de consommation de masse et de médiatisation de la décoration intérieure que le CAIM fera partie du Conseil du design français (CDF). Fondé par Paul Breton en 1969, le Conseil veut promouvoir le "design" auprès des pouvoirs publics. II est né sous l'impulsion d'associations engagées de longue date dans la création industrielle : la Chambre syndicale des Esthéticiens Industriels, et surtout Formes Utiles et l'Institut d'Esthétique industrielle. Cette fédération du design regroupe, sous un vocable peu consensuel à l'époque, l'Architecture intérieure, les arts appliqués, la Création de modèles, l'esthétique industrielle, le graphisme, le paysage et le stylisme. Mais les divisions de groupes auront raison de la pérennité et de l'efficacité de son action.

Le paradoxe est là : dans l'affirmation de compétences multiples, à la fois très spécialisées et généralistes, pour s'inscrire dans l'industrie et le cadre de vie contemporain, mais qui empêche de saisir clairement les spécificités nécessaires pour accéder à la reconnaissance statutaire et sociale. Les groupes professionnels
116. Société des Artistes Décorateurs (1961, p. 4).

117. Viénot (1951). 
118. Bulletin...(1977, p.4).

119. Le CAIM renommé Syndicat national des architectes d'intérieur (SNAI) puis Office de qualification des architectes d'intérieur (OPQAI) en 1981, est devenu en 2000 l'actuel Conseil Français des Architectes d'intérieur (CFAI).

120. À ce titre, les désignations utilisées par le décorateur Pierre Guariche sont intéressantes. Il fait d'abord partie d'un groupement en 1954 , l'Atelier de Recherches Plastiques (ARP) : création de modèles destinés aux logements de la Reconstruction. En 1957, suite à la dissolution du groupe, P. Guariche s'installe en tant que décorateur. Adhérent de l'ACMS, il utilise alors cette appartenance pour ces courriers ainsi que celle de "l'architecture intérieure". Ce n'est quand 1971 qu'il emploie le mot "design" avec la dénomination Bureau d'étude-Design et architecture, dénomination qu'il garde jusqu'à la fin de sa carrière libérale (Jacob, 2015).

121. Rappelons qu'il existe toujours notamment dans la fiche des métiers reconnus par 1'Institut National des Métiers d'art. (Disponible en: $\quad<$ https://bit. ly/2PAwrXJ>. Accès: $1^{\circ}$ nov. 2018).

122. Emery (1971, p.2). se positionnent les uns par rapport aux autres alors que leurs pratiques le plus souvent se chevauchent. Le malaise se perçoit dans le bulletin du CAIM de 1977 «Une profession encombrée et mal définie».

N'importe qui peut s'installer architecte d'intérieur ou décorateur, ou ensemblier décorateur, ou architecte décorateur, ou designer et dans l'esprit du public comme dans celui d'un certain nombre de professionnels (architectes, ingénieurs, promoteurs privés ou d'Etat, etc.l, les frontières entre les activités de ceux qui s'intitulent de telle ou telle façon sont mal connues. ${ }^{118}$

\section{Une reconnaissance professionnelle toujours confuse}

Le CAIM ${ }^{119}$ fonctionne comme un label de qualité et de modernité, une certification professionnelle indiquée sur les cartes de visite et entêtes de courrier. ${ }^{120}$ Malgré tout, le terme "décorateur", jusqu'à la fin des années 1960, résiste. Les pratiques professionnelles ne s'enferment pas dans des catégories ni dans les définitions syndicales. Certains décorateurs s'assimilent à l'antiquaire ou à l'architecte, à l'artiste ou à l'ingénieur-conseil. Pendant les années 1960, qu'il soit moderne ou éclectique là la manière de Madeleine Castaing, Emilio Terry ou Henry Samuel par exemple), le décorateur-ensemblier revêt différents visages. ${ }^{121}$ II se nomme parfois "designer" alors que la profession n'est pas règlementée. Au cours des années 1970, I'ajout d'un nouveau vocable (architecte d'intérieur-designer) ne clarifie en rien ses compétences au moment même où le design fonctionne lui aussi comme un concept flou. Marc Emery dans son éditorial de la revue L'architecture d'aujourd'hui en 1971 pose le problème en ces termes:

L'architecte se veut designer, mais le designer nie 'l'architecture'. Tous deux cherchent par des voies similaires une maîtrise globale de l'espace. Tous deux souffrent des mêmes confusions quant à la finalité de leur pratique et à leur insertion dans une société en mutation. ${ }^{122}$

Les termes anglicisés "design" et "designer", entrés dans le langage courant vers le milieu des années 1960, succèdent progressivement à "l'esthétique industrielle" et se banalise à la fin des années 1970. II est significatif d'observer le décalage entre la définition que souhaitent imposer les professionnels du "design industriel" et la réception sociale qui en faite. Malgré les efforts institutionnels du Centre de Création industrielle (CCI) né de l'initiative privée de I'UCAD en 1969 et du Conseil supérieur de la création d'esthétique industrielle (CSCEI), d'initiative publique en 1971 (dissout en 1975), le terme demeure rattaché à l'art, l'architecture, la décoration d'appartement, au "style" et à la "modernité". 
Aucune terminologie n'arrive à identifier clairement les pratiques de conception globale de l'environnement. Certes, de nouvelles désignations ne renvoient pas toujours à de nouveaux métiers. Elles traduisent avant tout de nouvelles dynamiques liées aux évolutions technique, sociale et commerciale. Les indéterminations terminologiques qui affectent les professionnels révèlent toutes les difficultés à saisir l'évolution de pratiques investies dans un champ d'applications plus élargi, se situant au carrefour de l'industrie, de l'art et de l'économie. Elles témoignent de l'absence de consensus au sein même des professions. En France, la légitimation culturelle de ces pratiques interdisciplinaires semble perpétuellement remise en cause. Aussi, I'histoire des institutions comme la Société d'Encouragement à l'Art et à I'Industrie (SEAI) ${ }^{123}$ et la SAD ${ }^{124}$ ou encore celle des relations entre associations de professionnels et pouvoirs publics ouvriraient de nouvelles voies à la compréhension des mécanismes en jeu dans la création, la diffusion et la réception des productions. Elle permettrait d'éclairer plus finement la construction des discours sur les pratiques et leur champ d'action, mais également de saisir le rôle spécifique des instances de légitimation dans la construction des figures d'artistes comme celle de l' "artiste décorateur".
124. Des comparaisons européennes sont possibles avec la Société suisse par exemple, l'Oeuvre (OEV), Fond d'archives PP 807, Archives cantonales vaudoises, Chavannes-prèsRenens (Suisse) 


\section{BIBLIOGRAPHIE}

ALBIN, Pierre. Avant Cannes, "Ensembliers » et reconstructeurs. L'Homme libre, Paris, n. 1987, 1 jan. 1922.

AMIC, Yolande. Le mobilier français 1945-1964. Paris: Editions du Regard, 1983.

ANONYME. L'idée de corporation (opinions de Auguste Rodin, Alexandre Charpentier, Eugène Grasset, Charles Christofle, Edmond Gustave Siot-Decauville, Henri Vever, Gustave Michel e Félix Gaudin). Art et Décoration, Paris, maio 1902. Supplément.

ANONYME. Touche à tout. out. 1910.

ANONYME. L'ensemblier. La vie parisienne, Paris, p. 757, 19 out. 1912.

ANONYME. Les oeuvres et les hommes. Le Correspondant, Paris, p. 1121, 1923.

BARRET, Maurice. Le Décor d'aujourd'bui, Paris, jun. 1935.

BAUDRILLARD, Jean. Le système des objets. Paris: Gallimard, 1968.

BELVILLE, Eugène. Lucien Bonvallet, décorateur. L'Art décoratif, Paris, v. 23, n. 136, p. 17-25, 1910.

BLONDEL, Jean-François. Cours d'architecture, ou traité de la décoration, distribution et construction des bâtiments. Paris: Desaint, 1777.

BOISSEAU, Émile-André. L'art décoratif au Salon des artistes français. Bulletin des Sociétés artistiques de l'est, Société lorraine des amis des arts, Nancy, p. 18, jan. 1912.

BONY, Anne. Les Années 50. Paris: Editions du Regard, 1983.

BRACQUEMOND, Felix. Du dessin et de la couleur. Paris: Charpentier, 1885.

BULlETIN DE LA SOCIÉTÉ DES ARTISTES DÉCORATEURS. Paris: Société des Artistes Décorateurs, n. 1, 1902. 
BULLETIN D'INFORMATION DU SYNDICAT NATIONAL DES CRÉATEURS D'ARCHITECTURES INTÉRIEURES ET DE MODÈLES. Paris: Syndicat National des Créateurs d'Architectures Intérieures et de Modèles, n. 2, oct. 1977.

CARDON, Émile. L'art au foyer domestique (la décoration de l'appartement). Paris: Renouard, 1884.

CLOUZOT, Henri. Les ensembles mobiliers. La Renaissance de l'art français et des industries de luxe, Paris, p. 349-362, ago. 1925.

COGNIAT, Raymond. Maurice Lafaille. Art et Décoration, Paris, abr. 1935.

D'AVILER, Charles Augustin. Dictionnaire d'architecture civile et bydrolique. Paris: C-H Jombert, 1755.

DICTIONNAIRE de la langue française. Paris: Littré, 1873-1874. tomo 2.

DUFRÊNE, Maurice. A propos de meubles : le siège ( $1^{\text {ère }}$ partie). Art et Décoration, Paris, $\mathrm{p}$. 21-31, $2^{\circ}$ semestre de 1913.

DUMONT, Jacques. Formes industrielles. La Maison Française, n. 165, março de 1963.

DUMONT, Marie-Jeanne. La SADG: histoire d'une societe d'architectes: premier e partie: 18771939. Paris: S.F.A, 1989.

EMERY, Marc. Editorial. L'architecture d'aujourd'bui, Paris, n. 155, abr./maio 1971.

EXPOSITION Internationale de Milan 1906, groupe VIII, classe 66 (Arts Décoratifs), Rapports de MM. Paris: Vermot, 1910.

FAVARDIN, Patrick. Les décorateurs des années 50. Paris: Norma, 2012.

FOREST, Dominique (Dir.). Mobi Boom, l'explosion du design en France, 1945-1975: catalogue d'exposition éponyme du 22 septembre 2010 au 2 janvier 2011. Paris: Les Arts Décoratifs, 2010.

FRAVALO, Fabienne. Le salon de l'art décoratif (1905-1906): exposer l'intérieur en vue d'un art total? Histoire de l'Art, n. 70, 2012. 
FROISSART-PEZONE, Rossella. L'Art dans tout: les arts décoratifs en France et l'utopie d'un Art nouveau. Paris: CNRS Editions, 2004.

FROISSART-PEZONE, Rossella. Les arts décoratifs au service de la Nation, 1880-1918: resumo do seminário de 17 de junho de 2005. 2005. Disponível em: <https://bit.ly/2Q66rjX>. Acesso em: 31 out. 2018.

GENCEY, Pierre. René Gabriel. Paris: Norma, 2016.

GENUYS, Charles. L'Exposition de la Société des artistes décorateurs. Art et Décoration, Paris, p. 78-95, jan. 1904.

GILLET, Louis. Le salon d'automne. Gazette des Beaux Arts, Paris, jul. 1921.

GUERINET, Armand. L'Art décoratif aux Salons de 1910: Société des artistes français, Société nationale des beaux-arts, Société des artistes décorateurs: bijoux, orfèvrerie, ornementation. Paris: Libraire d'art décoratif Armand Guérinet, 1910. prancha 24.

HAVARD, Henri. Dictionnaire de l'ameublement et de la décoration: depuis le XIIe siècle jusqu'à nos jours. Paris: Dourdan, E. Vial, 2012. tomo 2.

HÉRISSE, Émile. Manuel pratique du patissier-confiseur decorateur: a l'usage des chocolatiers, confiseurs, cuisiniers, glaciers. Paris: Létang Fils, 1894.

HERMANT, André. Formes utiles. Paris: Edition du Salon des Arts ménagers, Vincent Fréal et Cie, 1959.

IRIBE, Paul. Profits et pertes. Ameublement et Décoration, Paris, p. 5-7, 1931.

JACOB, Delphine. Pierre Guariche designer-architecte d'intérieur (1926-1995) et les nouveaux programmes issus de la croissance. 2015. Tese (Doutorado em História da Arte) - Université Paris 1, Paris, 2015.

JALOUX, Edmond. Lettre de Paris: le déclin du bibelot. La Revue Belge, Bruxelles, v. 2, n. 1, p. 460-463, avril 1934 .

JANNEAU, Guillaume. Introduction à l'exposition des arts décoratifs, considérations sur l'esprit moderne. Art et Décoration, Paris, 1925. 
JOURDAIN, Francis. Regards sur le monde du travail, Paris, 21 out. 1937.

LUNEAU, Jean-François. Art et industrie au XIXe siècle: des arts industriels aux industries d'art. In: LAMARD, Pierre; STOSKOPF, Nicolas (Org.). Art et industrie, XVIII-XIXe siècle: histoire industrielle et société. Paris: Picard, 2013. p. 21-22.

MAGNE, Lucien. L'art appliqué aux métiers, décor du verre, gobeletrie, mosaïque, vitrail. Paris: H. Laurens, 1913.

MAGNUSSON, Carl. La décoration intérieure au XVIIIe siècle: l'architecte et le sculpteur. Études de Lettres, Lausanne, n. 1, p. 1-13, 2009. Disponível em: <https://bit.ly/2OYiS50>. Acesso em: 6 jul. 2017.

MAIGNAN, Maurice. A propos du Salon des artistes décorateurs. L'Art Décoratif, Paris, n. 148, p. 192-202, abr. 1911.

MENEUX, Catherine. L'Art social au tournant du siècle. Arts E Sociétés, n. 12-1, 2006. Disponível em: <https://bit.ly/2Q9wgjf>. Acesso em: 31 out. 2018.

MICHEL, André. Artistes contemporains: Jean Dampt. La Revue de l'Art Ancien et Moderne, Paris, v. 34, n. 208, jul. 1914.

MOUTARD-ULDRY, Renée. Marcel Gascoin. Art et Décoration, Paris, v. 66, 1937.

NOUVEAU dictionnaire de la langue française. 3. ed. Paris: Larousse, 1856.

OLLIVIER, Carine. Les architectes d'intérieur: division du travail et concurrences. 2010. Tese (Doutorado em Sociologia) - Université de Versailles Saint Quentin en Yvelines, Versailles, 2010.

QUINCY, Quatremère de. Dictionnaire historique d'architecture: encyclopédie méthodique. Paris: Le septième salon des artistes décorateurs. Art et Industrie, 1832. tomo 1.

PILLARD-VERNEUIL, Maurice. Le salon des artistes décorateurs. Art et Décoration, Paris, p. 98-99, abr. 1914.

POSSEMÉ, Évelyne. 1910-1930: les années 25. Paris: Massin, 1999.

RENOUS, Pascal. Portraits de décorateurs. Revue de l'ameublement, Paris, 1969. 
ROCHES, Fernand. A propos des salons des 1911. L'Art Décoratif, Paris, v. 25, jun. 1911.

ROCHES, Fernand. Le Salon d'Automne de 1912. L'Art Décoratif, Paris, n. 184, nov. 1912.

ROYERE, Jean. De l'inutilité du décorateur. La Maison Française, Paris, v. 2, n. 16, p. 3, 1948.

SOCIÉTÉ DES ARTISTES DÉCORATEURS. Ata do comitê. maio 1901.

SOCIÉTÉ DES ARTISTES DÉCORATEURS. $1^{\text {ère }}$ exposition d'art décoratif, catalogue illustré.

Paris: Frazier-Soye, 1904.

SOCIÉTÉ DES ARTISTES DÉCORATEURS. Ata de 23 de janeiro de 1961. Arquivos, Paris, 1961.

VACHON, Marius. La crise industrielle et artistique en France et en Europe. Paris: Librairie illustrée, 1886.

VACHON, Marius. Pour la défense de nos industries d'art: l'instruction artistique des ouvriers en France, en Angleterre, en Allemagne et en Autriche. Paris: A. Lahure, 1899.

VAUXCELLES, Louis. Le Salon d'Automne de 1910. L'Art Décoratif, Paris, v. 23, out. 1910.

VAUXCELlES, Louis. Au Salon d'Automne, 2e article. L'Art Décoratif, Paris, 20 nov. 1911.

VAUXCELLES, Louis. Le septième salon des artistes décorateurs. Art et Industrie, Paris, abr. 1912.

VERA, André. La nouvelle architecture. L'Architecte, Paris, set. 1912.

VIÉNOT, Jacques. Esthétique industrielle. Paris : Éditions de Clermont, n. 1, 1951.

Artigo apresentado em 17/08/2017. Aprovado em 23/05/2018

\section{(cc) BY}

All the contents of this journal, except where otherwise noted, is licensed under a Creative Commons Attribution License 